\title{
Progesterone-induced Warburg Effect is Regulated by Cell-type-specific Interaction of Progesterone Receptor Membrane Component 1 and Hexokinases
}

Mohammad Golam Sabbir ( $\sim$ golam.sabbir@gmail.com )

Canadian Centre for Agri-Food Research in Health and Medicine, St. Boniface Hospital Research Centre, Winnipeg, MB, R2H 2A6, Canada. https://orcid.org/0000-0002-0943-2542

Carla G Taylor

University of Manitoba

Peter Zahradka

University of Manitoba

\section{Research}

Keywords: Progesterone, PGRMC1, Warburg effect, Phosphorylation, SUMOylation, Ubiquitination, Hexokinase, Mitochondria, Rough endoplasmic reticulum

Posted Date: August 20th, 2020

DOI: https://doi.org/10.21203/rs.3.rs-55518/v1

License: (c) (1) This work is licensed under a Creative Commons Attribution 4.0 International License. Read Full License 


\section{Abstract}

Background: Progesterone receptor membrane component 1 (PGRMC1) is a non-canonical progesterone (P4) binding protein. PGRMC1 is elevated in a variety of cancers and its phosphorylation state associated with hormone responsiveness in breast cancer. Metabolic reprogramming is a key factor for tumor growth during malignancies. Recently, we reported that the P4-inducedWarburg effectinHEK293cells is associated with altered post-translational modifications (PTMs) of PGRMC1, including phosphorylation, SUMOylation, and ubiquitination, which were linked to rapid proteasomal degradation of the protein. The previous study also identified hexokinase (HK) as a potential novel interacting partner of PGRMC1. HKs catalyze the first essential step of glucose metabolism and directly couple glycolysis to mitochondrial respiration. Therefore, in the present study, P4's effects on glycolysis and PTMs of PGRMC1 as well as its interaction with HKs were compared between HEK293 and HepG2 cells to unravel the signaling pathways that mediate cell-type-specific metabolic reprogramming.

Methods: P4-induced glucose metabolism in wild-type and PGRMC1-deficient cells wasassessed using the Seahorse flux analyzer, while PTMs of PGRMC1/HKs and protein-protein interaction were studied using immunoprecipitation, isoelectric focussing, phosphomimetics, and mass spectrometry.Translocation of HKs to different subcellular organelles were studied using subcellular fractionation, and the cell-type-specific effect of PGRMC1-deficiency on endoplasmic reticulum (ER) and mitochondria; ultrastructure were examined by electron microscopy.

Results:P4 treatment caused a rapid increase in glycolysis in HEK293 cells, whereas it decreased glycolysis in HepG2 cells. In addition, PGRMC1 was not degraded in HepG2 cells which is in contrast to HEK293 cells where rapid proteasomal degradation of PGRMC1 occurredfollowing P4 treatment. Besides, PGRMC1 half-life and PTMs under basal condition were found cell-type-specific and the P4-induced PTMsdiffered between the two cell types. Furthermore, we observed cell-type-specific interaction of HKs with PGRMC1, and differential translocation of HK1/2 to the ER, mitochondria and nuclear compartments following P4 treatment. PGRMC1 deficiency altered ER structure in HepG2 cells. Thus, multiple factors underlying the cell-type-specific P4-PGRMC1-mediated metabolic reprogrammingwere identified.

Conclusions: These findings provide a hitherto unknown novel P4-induced cell-type-specific PGRMC1-HK signaling mechanism that contributes to the molecular basis of P4-induced metabolic reprogramming, with important applications for hormone responsiveness in cancer.

\section{Background}

Progesterone receptor membrane component 1 (PGRMC1) is a non-classical membrane-associated progesterone receptor (MAPR) family protein [1], primarily expressed in the liver and kidney but also in other organs including brain, breast, heart, lung, and pancreas as well as reproductive tissues [1-3]. PGRMC1 regulates a variety of physiological processes to control cell proliferation and survival [4-9], lipid biosynthesis and metabolism $[10,11]$, homeostasis of iron and haem $[12,13]$, reproductive function [14- 
16], neurotoxicity [17], and stem cell self-renewal [18]. Upregulation and differential phosphorylation of PGRMC1 has been observed in a variety of cancers [19-23] including breast cancer which is known for its hormone responsiveness[24, 25]. Progesterone (P4) is a naturally occurring ligand for PGRMC1[26, 27]. P4 is synthesized from cholesterol by steroidogenic tissues in the gonads, placenta, adrenal cortex, and brain $[28,29]$ and regulates carbohydrate, lipid, and protein metabolism [30]. The role of P4-induced PGRMC1 signaling in cellular metabolism, specifically glucose catabolism, was not well known until it recently was reported that P4-PGRMC1 signaling causes rapid induction of aerobic glycolysis (Warburg effect) in human embryonic kidney-derived cells (HEK293), which was associated with rapid proteasomal degradation of a 75 kilodaltons $(\mathbf{k D a})$ PGRMC1 protein predominantly localized in the endoplasmic reticulum (ER) and mitochondria [3]. Native PGRMC1 is a $25 \mathrm{kDa}$ protein, but complex post-translational modification (PTM), via phosphorylation, SUMOylation, and ubiquitination, was found associated with the generation of the higher molecular mass of PGRMC1 (50-100 kDa) differentially located in several subcellular compartments [1, 3]. These findings led to the question: is the P4-induced and PGRMC1mediated Warburg effect an universal phenotype or a cell-type-specific metabolic phenomenon? Interestingly, P4-mediated carbohydrate metabolism is organ or tissue-specific; for example, P4 treatment causes glycogen catabolism in the uterine epithelium[31], whereas, it promotes glycogen storage in the liver [30] and inhibits glucose oxidation in skeletal muscle cells [32]. Besides, the P4-induced Warburg effect observed in HEK293 cells[3] is a key malignant adaptation[33], and sex hormones including P4 are associated with an increased risk of certain cancers[24, 25, 34]. Therefore, unraveling the mechanistic basis of cell-type-specific P4-PGRMC1 signaling and regulation of glucose metabolism is immensely important.

The classical seven-transmembrane P4 receptors $(\mathrm{mPRa} / \beta / \mathrm{Y} / \delta)$ were identified as putative $\mathrm{G}$ proteincoupled receptors (GPCRs)[35] and P4-induced signal transduction is involved in the activation of mitogen-activated protein kinases (MAPKs), inhibition of cAMP production and mobilization of intracellular $\mathrm{Ca}^{2+}$ ions $[35,36]$. In contrast, PGRMC1 is a single-pass transmembrane domain-containing protein[27] primarily localized in the endomembrane (endoplasmic reticulum, Golgi apparatus, and mitochondria ) $[3,37]$. The exact nature of P4-induced signal transduction through PGRMC1 is not known. A SUMOylated PGRMC1 has been shown to regulate P4-induced inhibition of T cell factor/lymphoid enhancer factor (TCF/LEF)-mediated transcriptional activity[38]. Further, it has been reported that extracellular signal-regulated kinase 5 (ERK5) and serpin 1 mRNA binding protein 1 (SERBP1) interact with PGRMC1 to regulate P4-induced secretion of brain-derived neurotrophic factor (BDNF) from glial cells [39] and the antiapoptotic effect in granulosa cells, respectively[40]. Thus, previous studies are not sufficient to provide a detailed mechanism for P4-PGRMC1 signaling-mediated regulation of cellular glucose metabolism. Interestingly, in a previous study, we identified by mass spectrometric (MS) analysis a variety of potential PGRMC1 interacting proteins in HEK293 cells including hexokinases (HKs) [3]. HKs are the first rate-limiting enzyme of glycolysis and the pentose phosphate pathway (PPP), whichcatalyzes the phosphorylation of glucose to glucose-6-phosphate (G6P) [41]. Four distinct isozymes of HK (HK1-4) have been identified in mammalian tissues [42], and they differ in substrate specificity, tissue-specific expression, and intracellular localization $[43,44]$. The HK1 and HK2 
isoforms are frequently referred to as mitochondrial HKs, as they differ from other isoforms by their unique capacity to directly interact with the voltage-dependent anion channel 1 (VDAC1) on the cytosolic side of the outer mitochondrial membrane (OMM) [44]. VDAC1 is a mitochondrial metabolite transporter that allows diffusion of small hydrophilic molecules including ADP/ATP [45]. Several experimental approaches led to the conclusion that mitochondrial HKs directly couple the first committed step of glucose metabolism to oxidative phosphorylation (OXPHOS)[43, 44]. During coupled respiration, the mitochondrial hexokinases selectively use intra-mitochondrial ATP as substrate, even if cytosolic ATP is available, and the ADP that is generated is channeled back into the mitochondria through VDAC1 to support OXPHOS[43, 44]. Such a feedback system ensures an overall rate of glucosemetabolism commensurate with cellular energy demandsand helps to avoid an excessive production of lactate due to the Warburg effect. Therefore, it is easy to envision that any alteration in the mitochondrial HKs may contribute to a metabolic shift which may provide a selective advantage to the cells, such as, for example, the highly glycolytic phenotype observed in the cancer cells. Taking these findings together, it was hypothesized that P4-induced PGRMC1 signaling-mediated rapid increase in glycolysis is cell-typespecific and linked to the interaction of PGRMC1 with mitochondrial HKs.

Human Protein Atlas (HPA)-based tissue expression profiles have shown that PGRMC1 is highly expressed in the liver [46]. Therefore, wild-type and PGRMC1-depleted HEK293 and hepatoma-derived HepG2 [47] cells were used to perform a comparative evaluation of P4-induced glucose metabolism as well as study the interaction of PGRMC1 with HKs. Furthermore, P4-induced PTMs and proteasomal degradation of PGRMC1, and the intracellular distribution of both PGRMC1 and HKs, were compared between the two cell types to examine the underlying mechanism of P4-induced metabolic reprogramming.

\section{Methods}

\section{Cell culture}

The HEK293 and HepG2 cells were cultivated in Dulbecco's modified Eagle's medium (DMEM) supplemented with $10 \%$ heat-inactivated FBS and $1 \times$ antibiotic antimycotic solution (A5955, Sigma). The CRISPR/Cas9-mediated PGRMC1 knockout HepG2 cells were generated as described previously[3].

\section{Isolation and enrichment of endoplasmic reticulum (ER)/mitochondrial fraction}

Mitochondria were isolated by a method previously described $[3,48]$. Briefly, 80-90\% confluent cells were washed with PBS and harvested in mitochondrial stabilization buffer (MSB-1) containing $70 \mathrm{mM}$ sucrose, $210 \mathrm{mM}$ mannitol, $5 \mathrm{mM}$ HEPES pH 7.2, and $1 \mathrm{mM}$ EGTA. The cells were disrupted with a Teflon Dounce homogenizer and the homogenate was centrifuged at $800 \mathrm{~g}$ for $10 \mathrm{~min}$ at $4^{\circ} \mathrm{C}$. Following centrifugation, the supernatant was decanted through 2 layers of cheesecloth into a separate tube and centrifuged at $8000 \mathrm{~g}$ for $10 \mathrm{~min}$ at $4^{\circ} \mathrm{C}$. After removal of the supernatant, the pellet was resuspended in MSB- 1 and the 
centrifugation was repeated. The final pellet was resuspended in lysis buffer and used for immunoblotting.

\section{Western blotting and immune-detection and quantification}

Relative quantification of proteins was done by SDS-PAGE-based separation of total proteins followed by nitrocellulose membrane transfer and immunoblotting-based detection using HRP-conjugated secondary antibodies. The detailed methodology for the relative quantification of the proteins-of-interest in the immunoblots has been previously described in detail [48]. Table 1 lists the primary antibodies used in this study. The cell lysates were prepared in 1× RIPA lysis and extraction buffer (ThermoFisher Scientific, Cat No: 89900 ) supplemented with $1 \times$ Halt protease and phosphatase inhibitor cocktail (ThermoFisher Scientific, Cat No: 78441). The protein lysates were denatured in Laemmli buffer containing $2 \%$ SDS, $10 \%$ glycerol, $0.002 \%$ bromophenol blue, and $0.75 \mathrm{M}$ Tris-HCl pH 6.8 supplemented with $100 \mathrm{mM}$ DTT. The immunoblots were quantified using ImageJ (version 1.48) Software[49].

\section{RNA extraction, cDNA synthesis, and multiplex reverse-transcription PCR (RT-PCR)}

Total RNA $(1-5 \mu \mathrm{g})$ extracted by Trizol reagent (ThermoFisher Scientific, Canada, Catalog number: 15596018) was treated with RNase-free DNase I (New England Biolabs Inc., Catalog number: M0303) at $37 \otimes C$ for $15 \mathrm{~min}$, subsequently heat-inactivated at $75^{\circ} \mathrm{C}$ for $10 \mathrm{~min}$ and used for CDNA synthesis. The firststrand cDNA was synthesized using the SuperScript ${ }^{\mathrm{TM}}$ first-strand synthesis system (ThermoFisher Scientific, Canada, Catalog number: 11904018). Multiplex RT-PCR and regular RT-PCR were performed in a $20 \mu \mathrm{L}$ reaction mix containing $1 \times$ buffer, $2 \mathrm{mM}$ dNTP mix, $0.2 \mu \mathrm{M}$ oligonucleotide primers, cDNAs equivalent to $100 \mathrm{ng}$ total RNA, and 1.25 units KOD Hot Start DNA Polymerase (Millipore Sigma, Catalog number: 71086)/DreamTaq ${ }^{\text {TM }}$ Hot Start DNA Polymerase (ThermoFisher Scientific, Catalog number: EP1701), respectively, and amplified using $98 / 95^{\circ} \mathrm{C}$ for $1 \mathrm{~min}, 35$ cycles of $95^{\circ} \mathrm{C}$ for $10 \mathrm{sec}, 55^{\circ}-65^{\circ} \mathrm{C}$ for $10 \mathrm{sec}$ and $72^{\circ} \mathrm{C}$ for $90 / 30 \mathrm{sec}$. The RT-PCR products were separated using agarose gel electrophoresis and visualized.

\section{Seahorse flux analyzer-based glycolysis assay}

Oxygen consumption rate (OCR) and extracellular acidification rate (ECAR) were measured simultaneously using a Seahorse Biosciences XF24 analyzer (Agilent) as previously described $[3,48]$. Cultured cells were grown in 24 well assay plates overnight. Cells were then washed thrice in pre-warmed serum free XF assay medium (non-buffered DMEM) supplemented with $1 \mathrm{mM}$ sodium pyruvate ( $\mathrm{pH} 7.4)$. In the glycolysis stress test, an appropriate volume of glucose-free pre-warmed XF assay media was added into the individual wells of the XF24 analyzer plate and incubated in a non- $\mathrm{CO}_{2}$ incubator for 1 hour. Pre-warmed glucose $(10 \mathrm{mM})$, oligomycin $(1 \mu \mathrm{M})$ and 2-deoxyglucose $(50 \mathrm{mM})$ were added sequentially into the injector ports $A, B$ and $C$ of the sensor cartridge, respectively. The concentrations mentioned are final concentrations. The data were analyzed using WAVE software (Agilent Technologies, version 2.6.0). 


\section{Site-directed mutagenesis and plasmid transfection}

The N-terminal Flag-PGRMC1 ORF cloned in the pcDNA3.1+/C vector was obtained from GenScript (Catalog: OHu21154). The Flag-PGRMC1 plasmid was used to create S57A, S57D and S57E mutants using a Q5 site-directed mutagenesis kit (New England Biolabs Inc. Catalog number: E0554S) and the primer sequences listed in Table-1. The plasmids were sequenced using T7 and Sp6 sequencing primers to verify the desired mutations. The phosphomimic mutant plasmids were transfected using Lipofectamine 3000 reagent (Thermo Fisher, catalog number:L3000001).

\section{Immunoprecipitation of Flag-PGRMC1 and mass spectrometry}

The $\mathrm{N}$ terminal Flag tagged PGRMC1 plasmid was transfected into HEK293 and HepG2 cells and the cells were serum starved for 24 hours. The cells were lysed using $1 \times$ phosphate buffered saline (PBS) supplemented with 1\% IGEPAL-CA630 (Sigma, Cat. No: 18896) and 1.5\% n-Dodecyl $\beta$-D-maltoside (Sigma, Cat. No: D4641). The Flag-PGRMC1 was then pulled from total cellular lysates using Bs3-conjugated (bissulfosuccinimidyl-suberate, ThermoFisher, Cat. No-A39266) as well as unconjugated Dynabeads-protein G-anti-Flag antibody. Dynabeads-protein G (ThermoFisher, Cat. No: 10004D) was used as a negative control in the immunoprecipitation. The immunoprecipitated Flag-PGRMC1 complex was denatured, reduced, alkylated and precipitated. The precipitated proteins were dissolved in $6 \mathrm{M}$ urea, with volume adjusted using $20 \mathrm{mM}$ Tris buffer ( $\mathrm{pH} 8.0)$, and digested overnight at $37^{\circ} \mathrm{C}$ on a shaking platform using a Trypsin/Lys-C protease mixture (Promega, Cat. No: V5071). The digested peptides were desalted using Pierce C-18 $100 \mu$ lips (ThermoFisher Scientific, Cat. No: 87784) and analyzed by tandem mass spectrometry (MS) analysis using the AB SCIEX TripleTOF ${ }^{\mathrm{TM}} 5600$ System (Applied Biosystems/MDS Sciex, Foster City, CA) at the Manitoba Centre for Proteomics and Systems Biology, University of Manitoba.

\section{Isoelectric focusing (IEF)}

Fifty microgram of total cell lysate protein was precipitated by acetone and dissolved in rehydration buffer containing $8 \mathrm{M}$ Urea, 2\% CHAPS, $50 \mathrm{mM}$ dithiothreitol (DTT) and 0.2\% Bio-Lyte ampholytes (pH 310). The dissolved proteins were then incubated in Zoom IPG 3-10 nonlinear (NL) or Linear (L) strips (ThermoFisher Scientific) for $1 \mathrm{~h}$ and focused at 175 volt (V) for $15 \mathrm{~min}, 175-2000 \mathrm{~V}$ ramp for $45 \mathrm{~min}$ and $2000 \mathrm{~V}$ for $30 \mathrm{~min}$. After focusing, the proteins in the strips were reduced (by $130 \mathrm{mM} \mathrm{DTT}, 15 \mathrm{~min}$ ) and alkylated (by $135 \mathrm{mM}$ iodoacetamide, $15 \mathrm{~min}$ ) while in an equilibration buffer containing $6 \mathrm{M}$ Urea, $2 \%$ SDS, $0.375 \mathrm{M}$ Tris ( $\mathrm{pH} 8.8$ ) and $20 \%$ glycerol. The proteins were then resolved on second dimension SDSPAGE and immunoblotted.

\section{Transmission electron microscopy (TEM)}

ForTEM, cells were grown on Matrigel coated $2 \mu \mathrm{m}$ nitrocellulose membranes [50] and kept in medium without serum for 24 hours. Alternatively, the ER/mitochondrial fractions were precipitated as a pellet 
onto a nitrocellulose membrane. The cells/pellets were then fixed with $2 \%$ glutaraldehyde in Sorenson's buffer $(\mathrm{pH} 7.4)$ at $4^{\circ} \mathrm{C}$ for 2 hour. Following fixation, the membranes were dehydrated and finally embedded in epoxy resin and polymerized at $60^{\circ} \mathrm{C}$ overnight. Sections were cut on a Leica EM UC7 ultramicrotome; at first, semi-thin sections $(0.5 \mu \mathrm{m})$ were stained with toluidine blue and examined under light microscopy to identify the area of interest and confirm the tissue orientation. Ultrathin sections $(70 \mathrm{~nm})$ were then transferred to copper grids (Ted Pella Inc), and stained with uranyl acetate and lead citrate, and examined on a Phillips CM 100 Compustage (FEI) transmission electron microscope and digital micrographs were captured with an AMT CCD camera (Deben).

\section{Statistical analysis}

Statistical analysis was performed using Prism version 7.00 (GraphPad Software). The means of more than 2 groups were compared using one-way ANOVA (randomized) [51, 52]. followed by Dunnett's posthoc multiple comparison test to compare the means of multiple experimental groups against a control group mean [51]. Comparisons between two groups were performed using Student's t test (unpaired). Differences were considered significant with $\mathrm{P}<0.05$.

\section{Results}

\section{Primary adult human tissue-specific expression of PGRMC1.}

Tissue-specific expression of PGRMC1 was studied by reverse transcription PCR (RT-PCR) using primary adult human adipose, artery, bone marrow, cerebellum, cortex, intestine, liver, smooth muscle, and skin tissue derived RNAs. A set of primers was designed to amplify the full-length PGRMC1 open reading frame (Table-1, Fig 1A). Long-range RT-PCR amplification using cDNAs equivalent to $100 \mathrm{ng}$ total RNA revealed the presence of an expected band corresponding to the full-length PGRMC1 amplicon in the adipose, artery, cortex, intestine, liver, skeletal muscle and skin tissues (Fig 1B). Based on this expression profile, the choice of hepatoma-derived HepG2 [47] cells as a model system to study P4-PGRMC1 signaling-induced glucose metabolism was justified [3].

\section{Seventy kDa PGRMC1 is abundant in the ER and mitochondrial fractions in HepG2 cells.}

Immunoblotting using an anti-PGRMC1 mouse monoclonal antibody (Table 1) revealed the presence of p25, p70, and p90-110 PGRMC1 in HepG2 cells grown overnight in serum-supplemented as well as serum-deprived media (Fig 1C). In HepG2 cells, p70 PGRMC1 is the predominant form as previously observed in HEK293 cells[3]. A non-detergent-based cell fractionation method previously used to separate cytosol, enriched endoplasmic reticulum (ER)/mitochondria, and nuclear fractions in HEK293 cells [3] and EA.hy926 endothelial cells[48] was used to fractionate/enrich subcellular organelles in HepG2 cells (Fig S1A). Both immunoblotting, as well as transmission electron microscopy (TEM), were used to analyze HEK293 and HepG2 cell structures and the nature and enrichment of subcellular fractions. HepG2 cells were grown on the nitrocellulose membrane to study the cellular ultrastructure by TEM (Fig 1E). TEM images revealed the presence of elongated tubular rough endoplasmic reticulum (RER) in the HepG2 cells 
(Fig 1F). The majority of the mitochondria in HepG2 cells were found in contact with RER and arranged in an orderly manner sandwiched between the tubules of RER (Fig 1F, yellow arrow). Following initial homogenization, the resultant subcellular fraction was designated as fraction-1 (F1), which was enriched in RER and mitochondrial structures (Fig $1 \mathrm{GH}$ ). The nuclear fraction was found with perinuclear ER and mitochondrial structures (Fig S1A-C, red dotted areas in BC). Therefore, the nuclear fraction was treated with a mild non-ionic detergent ( $0.5 \%$ NP40) to dissociate the attached perinuclear organelles (Fig S1A). The detergent treatment resulted in a smooth rounded nuclear fraction which was devoid of any residual $\mathrm{ER} /$ mitochondrial structures (Fig 1I). Based on this procedure and findings, the mild non-denaturing detergent-extracted perinuclear ER/mitochondrial fraction was designated as fraction 2 (F2). Next, a series of subcellular organelle-specific protein markers were used to validate the fractionation process and to identify PGRMC1 intracellular distribution (Fig 1J-0). a-Tubulin (TUB1A), calnexin (CANX) [53], VDAC1 [54], and histone-H3 (H3) were used as a cytosolic, ER, mitochondria, and nucleus-specific markers, respectively (Fig $1 \mathrm{~J}-\mathrm{N}$ ). The Oriole-stained gel indicated considerable differences between the total protein profiles of different subcellular fractions (Fig 10) which provided additional support for the efficiency of the enrichment process. The p70 PGRMC1 was found relatively abundant in the $\mathrm{ER} /$ mitochondrial fraction compared to the nucleus (Fig 1J). The p25 PGRMC1 was not detectable in the subcellular fractions (within detection limit). The p90-100 PGRMC1 was observed as faint bands following longer exposure of the immunoblots and, therefore, it was not shown. Overall, p70 is the dominant form of PGRMC1 in HepG2 cells as previously observed in HEK293 cells[3]. Expression of an Nterminal Flag-tagged PGRMC1 in HepG2 cells indicated localization of Flag-PGRMC1 in the ER (Fig S2A, yellow arrows). Flag-PGRMC1 appeared as a p25, p50, and >p75 proteins (Fig S2B-C).

\section{P4-induced Warburg effect is cell-type-specific.}

P4-induced cellular glucose metabolism was interrogated by simultaneous measurement of oxygen consumption rate (OCR) and extracellular acidification rate (ECAR) using the Seahorse flux analyzer as described previously[3]. ECAR approximates glycolysis and OCR is an important metric for mitochondrial function $[3,55]$. The glycolysis stress test [56] was performed to study the P4-induced Warburg effect. In the glycolysis stress test, the glucose-induced response is reported as the rate of glycolysis under basal conditions (Fig 2A, grey-colored area). ECAR, prior to glucose injection, is referred to as non-glycolytic acidification which is caused by processes in the cell other than glycolysis (Fig. 2A, yellow-colored area). The injection of oligomycin, an ATP synthase inhibitor, inhibits mitochondrial ATP production, and shifts energy production to the glycolysis. A subsequent increase in ECAR reveals maximum glycolytic capacity in the cell (Fig. 2A, cyan area). Finally, injection of 2-deoxyglucose (2-DG), a glucose analog that inhibits glycolysis through competitive binding to HKs, the first enzyme in the glycolytic pathway, resulted in a decrease in ECAR and confirmed that ECAR produced in the experiment is due to glycolysis (Fig. 2A). As reported previously[3], treatment of HEK293 cells with $10 \mu \mathrm{M}$ P4 caused a significant increase in basal glycolysis and a concomitant decrease in respiration within 30-40 min of the treatment compared to vehicle-treated cells (Fig. 2AB). In contrast, the P4-induced glycolysis as well as respiration was found to be significantly decreased in HepG2 cells (Fig 2AB). Previously, the dose-dependent analysis revealed that 10 MM P4 elicited a maximum glycolytic response in HEK293 cells within 30 min of treatment[3]. 
Therefore, we performed a dose-dependent analysis in HepG2 cells which revealed that $10 \mu \mathrm{M}$ P4 is also the most effective concentration to elicit a significant alteration in glycolytic response (Fig S2D). Thus, the use of $10 \mu \mathrm{M}$ P4 to compare the P4-induced Warburg effect between HEK293 and HepG2 cells was justified. Interestingly, oligomycin treatment increased glycolysis (glycolytic reserve volume) in vehicletreated HEK293 cells but not in HepG2 cells (Fig 2A). In contrast, P4-treatment caused a significant increase in the glycolytic reserve volume in HEK293 cells, whereas the effect was marginal in HepG2 cells (Fig 2C). Overall, these results indicate that the P4-induced Warburg effect is cell-type-specific.

In contrast to the cell-type-specific differential effect of $\mathrm{P} 4$ on glycolysis, cellular respiration rate (OCR) was found significantly decreased in both cell types within 30 min after P4 treatment (Fig 2D-E). Thus, it is evident that P4-mediated regulation of glycolysis and respiration may be independent events.

\section{P4-induced proteasomal degradation of PGRMC1 is cell type-specific.}

Previously, it was demonstrated that $10 \mu \mathrm{M} \mathrm{P4}$ caused rapid (within 30 min of treatment) proteasomal degradation of p70 PGRMC1 in HEK293 cells, which was inhibited with a proteasome inhibitor [3]. Based on this, it was concluded that P4-induced PGRMC1 degradation and the Warburg effect are physiologically connected events. Therefore, it was expected that PGRMC1 would not be degraded in HepG2 cells as it has exhibited the opposite effect in P4-induced glycolysis compared to HEK293 cells (Fig 2A-C). As anticipated, immunoblotting revealed that p70 PGRMC1 is not degraded in HepG2 cells within 30 min to 1.5 hours after P4 treatment compared to the HEK293 cells (Fig 2FG). Immunoblottingbased quantification revealed a significant decrease in p70 PGRMC1 levels in HEK293 cells (Fig 2H) whereas, it remained unaltered in HepG2 cells during the course of P4-treatment (Fig 2I). Thus, it is likely that the P4-induced Warburg effect and proteasomal degradation of PGRMC1 are interconnected and cell-type specific.

\section{PGRMC1 half-life is cell-type-specific.}

Turnover and half-life of a protein are dependent on the synthesis and degradation rate in the cell [57]. Therefore, P4-induced cell-type-specific differential proteasomal degradation of PGRMC1 may be associated with a potential difference in the half-life of PGRMC1 in HEK293 and HepG2 cells. One of the methods to determine the turnover rate of a protein is to use cycloheximide to block protein synthesis and the natural decay of the protein over time is measured by immunoblotting [58]. We treated HEK293 and HepG2 cells with $50 \mu \mathrm{g} / \mathrm{ml}$ cycloheximide and examined the PGRMC1 level at different time points (Fig S3). Immunoblotting revealed that half-life of p70 PGRMC1 in HEK293 cells is 2 hours (Fig S3AB) whereas, it is 5-6 hours in HepG2 cells (Fig S3CD). Thus, the cell-type-specific difference in PGRMC1 halflife is a factor underlying the P4-induced bioenergetic differences.

\section{PGRMC1 deletion in HepG2 cells failed to reverse the P4-induced decrease in glycolysis.}

Multiple independently selected PGRMC1 knockout $\left(\mathrm{PGRMC}^{-/}\right.$) HepG2 cell clones were generated using CRISPR/Cas9-mediated genetic ablation of PGRMC1 (Fig 3A-H). Both exon 2 and exon 3 of PGRMC1 
were targeted for the homology-driven insertion of a floxed reporter/selection cassette (Fig 3A) which was subsequently deleted by transient Cre-recombinase expression in HepG2 cells. Immunoblotting revealed the loss of PGRMC1 protein in the PGRMC ${ }^{-/}$HepG2 cell clones (Fig 3B). Interestingly, the level of ERspecific CANX protein was found to be significantly reduced in $\mathrm{PGRMC}^{-/-} \mathrm{HepG} 2$ cell clones compared to wild-type (Fig 3CD). Also, the total protein profiles of the PGRMC ${ }^{-/-} \mathrm{HepG} 2$ cell clones were visually different from that of wild-type cells indicating a global difference in the abundance of different species of proteins (Fig 3E). The loss of PGRMC1 in knockout clones was further validated by long-range RT-PCR which revealed the absence of the PGRMC1 ORF in the selected clones, thus indicating complete deletion of the gene (Fig 3F). HepG2 cells express a paralogous PGRMC2 gene (Fig 3G). Therefore, we used multiplex RT-PCR with primers specific to PGRMC1 \& PGRMC2 to simultaneously co-amplify both genes in a single reaction. Multiplex RT-PCR revealed the selective loss of PGRMC1 expression in the PGRMC1 1 HepG2 cell clones, whereas, PGRMC2 expression remained unaltered, thus showing that this targeted deletion approach selectively affected PGRMC1 (Fig 3H). In subsequent experiments, four independently

selected PGRMC1 ${ }^{-/-}$HepG2 cell clones were used to eliminate clonal variation. Previously, in HEK293 cells, genetic ablation of PGRMC1 inhibited the P4-induced Warburg effect [3], therefore, it was expected that loss of PGRMC1 in HepG2 cells would inhibit P4-induced inhibition of glycolysis. Interestingly, the loss of PGRMC1 failed to inhibit the P4-induced decrease in glycolysis observed in wild type HepG2 cells (Fig 3IJ). Further, PGRMC1 loss showed no effect on the P4-mediated decrease in cellular respiration (Fig $3 K L)$. Overall, these results indicate that HEK293 and HepG2 cells differed in terms of reversal of the P4 induced effect on glycolysis under PGRMC1 loss-of-function conditions.

\section{PGRMC1 regulates ER distribution and ER-mitochondrial contacts in HepG2 cells.}

The ER and mitochondria are structurally and functionally linked thorough contact points defined as mitochondria-associated ER membranes (MAM), which play a key role in cellular metabolism by controlling mitochondrial dynamics and function[59, 60]. Therefore, the ultrastructure of MAM was studied in HepG2 and compared with HEK293 cells to understand the cell type-specific differences in P4induced glucose metabolism. The ultrastructure of HEK293 cells was studied previously[3]. The TEMbased study using HepG2 cells revealed the presence of abundant elongated tubular RER in the wild-type cells (Fig 1F, S4A, yellow arrows). In contrast, fragmented RER was observed in PGRMC1 ${ }^{-/-}$HepG2 cells (Fig S4B, yellow arrows). Furthermore, the orderly distribution of mitochondria appearing as sandwiched between tubules of RER and the frequent contacts between these organelles (MAM) was lost in $\mathrm{PGRMC1}^{\%-}$ HepG2 cells compared to the wild-type (Fig S4CD, green arrows).

In HEK293 cells, the ER and MAM structures are not as abundant as compared to the HepG2 cells (Fig 1, S4, and S5). The ultrastructure of HEK293 cell was previously reported[3], however, it is presented in this study to compare with the HepG2 cells. Also, loss of PGRMC1 in HEK293 cells caused no visibly discernible dramatic effect on ER and mitochondrial distribution or MAM structures compared to the HepG2 cells (Fig S4 and S5). In addition, TEM analysis of the enriched ER/mitochondrial fractions from both cell types exhibited relatively less frequent MAMs in HEK293 compared to the HepG2 cells (Fig S6, 
yellow arrows). Overall, HepG2 and HEK293 cells are dramatically different in terms of organization of the ER and MAM structures, and loss of PGRMC1 severely altered structural organization of the ER and MAM in HepG2 cells compared to the HEK293 cells.

\section{Post-translational modifications of PGRMC1 are cell type-specific.}

In the previous study [3], PGRMC1 was found to be phosphorylated (P), acetylated (Ac), SUMOylated, and ubiquitinated in HEK293 cells. MS analysis identified P-S57, P-Y107, P-Y180, and Ac-K136 residues in PGRMC1. It was also found that P4-treatment caused a significant reduction in P-PGRMC(Ser) in HEK293 cells. Therefore, we studied the PTMs of PGRMC1 in HepG2 cells and compared them with HEK293 cells. Flag-PGRMC1 was transiently-expressed in HepG2 cells and immunoprecipitated. Immunoblotting revealed the presence of p25, p63, p100, and p>245 kDa PGRMC1 in the anti-Flag immunoprecipitate (Fig 4A-B). Immunoblotting of the Flag-PGRMC1 immunoprecipitate using anti-P-Ser, anti-SUMO2/3/4, and anti-ubiquitin antibodies revealed that Flag-PGRMC1 protein in HepG2 cells is phosphorylated, SUMOylated, and ubiquitinated (Fig 4C-E). Next, we performed MS analysis of the Flag-PGRMC1 and identified P-T178, P-Y180, and P-S181 residues of PGRMC1 in HepG2 cells (Fig 4F-I, Supplementary Table 1). The PTMs (phosphorylation and acetylation) of PGRMC1 differed in HEK293 and HepG2 cells as summarized in Figure 4F. The difference in phosphorylation is highlighted in the crystal structure of PGRMC1 in Figure 5GH. The P-S47, P-Y107, and Ac-K136 residues of PGRMC1, previously identified in HEK293 cells, were not detected in HepG2 cells, however, corresponding non-phosphorylated or nonacetylated peptide fragments were detected in the MS analysis (Fig 4I). The detection of P-Ser positive bands in the Flag-PGRMC1 immunoprecipitate validated this MS-based detection of P-PGRMC1(S181) in HepG2 cells, while the ubiquitination and SUMOylation of PGRMC1 provided an explanation for the higher molecular mass forms of the protein. Single ubiquitination or SUMOylation adds approximately 10 $\mathrm{kDa}$ or $8.6 \mathrm{kDa}$ molecular mass to a protein, respectively; therefore, it is possible that either multiple residues are involved or there may be poly-ubiquitination/SUMOylation [3].

\section{P4-induced post-translational modification of PGRMC1 is cell type-specific.}

In the previous study, it was found that P4 treatment of HEK293 cells caused a significant alteration in PGRMC1 PTMs (phosphorylation, SUMOylation, and ubiquitination) [3]. Therefore, the Flag-PGRMC1 immunoprecipitate from P4 and vehicle (ethanol)-treated HepG2 cells were subjected to immunoblotting using anti-P-Ser, anti-SUM02/3/4, and anti-ubiquitin antibodies (Fig 5A-F). A faint p25, a p60, and a strong p100 PGRMC1 bands were detected in both P4 and vehicle (ethanol)-treated immunoprecipitate (Fig 5A-C). The p100 PGRMC1 reacted positively for anti-P-Ser (Fig 5D), anti-SUMO2/3/4 (Fig 5E), and anti-ubiquitin (Fig 5F) antibodies. In contrast to HEK293 cells [3], Image J-based analysis of the immunoblots revealed no significant difference in the PTMs of PGRMC1 in P4 treated and vehicle-treated HepG2 cells. It is possible that the pan anti-P-Ser antibody may not detect all phosphorylated epitopes of PGRMC1 in HepG2 cells. Therefore, total proteins from the P4 and vehicle-treated Flag-PGRMC1 overexpressed HepG2 (Fig 5G-I) and HEK293 (Fig 5J-L) cells were subjected to isoelectric focusing (IEF) followed by SDS-PAGE and immunoblotting. IEF/SDS-PAGE analysis revealed the presence of pHख8-9 
(blue rectangle), $\mathrm{pH} \otimes 6-7$ (green rectangle), and $\mathrm{pH} \otimes 5-6$ (red rectangles) fractions of PGRMC1 containing p60, p75 and p>75 proteins, each corresponding to a specific isoelectric point (pl) and molecular mass representing different PTMs in HepG2 cells (Fig 5GI). A comparison between the charged fractions of PGRMC1 revealed that P4 treatment caused the disappearance of p75 (pI\&8-9) fraction (green arrows) and shifted p60 (plष5-6) fraction (red/white arrows) towards more negative pl/pH (Fig 5G-I). IEF/SDSPAGE using the P4 and vehicle-treated HEK293 cell-derived total proteins revealed the presence of pH『910 (blue rectangle), $\mathrm{pH} \otimes 8-9$ (green rectangle), and $\mathrm{pH} \otimes 3-4$ (red rectangles) fractions of PGRMC1 containing p60 and p75 proteins (Fig 5JK). A comparison revealed that P4 treatment caused the disappearance of a fraction of p60 (plष8-9) PGRMC1 (black arrows) and reduced the abundance of the p60 (pl『3-4) fraction (Fig 5J-L blue \& white arrows) compared to the vehicle-treated cells. Thus, it is evident that the basal (vehicle-treated), as well as P4-treated, PTMs of PGRMC1 are cell-type-specific which may account for the differential effect of P4 on glucose metabolism in the different cells.

\section{Cell-type-specific interaction of PGRMC1 and HKs.}

To validate potential cell-type-specific interaction of PGRMC1 and HKs, and to study the effect of P4 on such interaction, we subjected a Flag-PGRMC1 immunoprecipitate from the vehicle and P4-treated HEK293 and HepG2 cells to immunoblotting using anti-HK1 and -2 antibodies. Immunoblotting revealed the presence of a p25, p60, and p100 PGRMC1 in flag-PGRMC1 transiently expressed HEK293 cells (Fig $6 A B$ ). The absence of IgG light and heavy chain specific bands in the Oriole/Coomassie-stained gels (Fig 6A) was due to the chemical (BS3) crosslinking of the primary antibodies with the protein $\mathrm{G}$ beads. Immunoblotting of the Flag-PGRMC1 immunoprecipitate from the vehicle and P4-treated HEK293 and HepG2 cells revealed the presence of a p100 HK1 in both cell-types (Fig 6C-F). Increased exposure of the immunoblots revealed a p135 HK1 associated with both vehicle and P4 treated HEK293 cells (Fig 6D). In contrast to HEK293 cells, multiple $p \geq 135$ HK1 proteins were found in HepG2 cells to associate with P4treated PGRMC1, whereas, only p100 HK1 associated with PGRMC1 in the vehicle-treated cells (Fig 6F). Interestingly, a p100 HK2 was found associated with PGRMC1 in HEK293 cells but not in HepG2 cells (Fig $6 \mathrm{GH})$. Overall, these results indicate a cell type-specific differential interaction between PGRMC1 and HKs which may be responsible for the observed difference in the P4-induced metabolic reprogramming.

\section{HK2 is differentially modified in HEK293 and HepG2 cells.}

High throughput mass spectrometry analysis documented multiple phosphorylation and ubiquitination sites at functionally relevant residues in HK1 and HK2 archived in the PhosphositePlus database (Fig 6IJ) $[55,61]$. Differential PTMs of HK2 may be responsible for the observed loss of interaction with PGRMC1 in HepG2 compared to the HEK293 cells. Therefore, two-dimensional IEF/SDS-PAGE analysis was performed to study the charged PTM fractions of HK2 in HEK293 and HepG2 cells. HK2 appeared as several charged fractions within the $\mathrm{pH} 4-5$ region in vehicle-treated HEK293 cells (Fig 6K, red rectangle); these fractions shifted to a more negative $\mathrm{pH}$ in the P4-treated cells (Fig 6K, white rectangle). In contrast, $\mathrm{HK} 2$ appeared as several charged fractions within the $\mathrm{pH} 4-5$ (red rectangle) and $\mathrm{pH}$ 8-10 (blue rectangle) regions in the vehicle-treated HepG2 cells (Fig $6 \mathrm{~L}$ ), which shifted to the more negative $\mathrm{pH} \otimes 3-4$ 
region in the P4-treated cells (Fig $6 \mathrm{~L}$, yellow rectangle). It is interesting to note the HK2 $\mathrm{pH} 8-10$ fractions (blue rectangle) were not detected in the HEK293 cells (Fig 6K) compared to HepG2 cells (Fig 6L). Also, the $\mathrm{pl} / \mathrm{pH}$ shift in the charged fractions of HK2 in the P4-treatment HEK293 and HepG2 cells was not identical in the two cell types (Fig 6KL, white rectangles in the overlaid images at the bottom panel). Thus, differential PTMs may be responsible for the loss of interaction between HK2 and PGRMC1 in HepG2 cells compared to HEK293 cells. Identifying these modifications of HK2 is beyond the scope of this study.

\section{Phosphomimics revealed that S57 phosphorylation is not responsible for cell type-specific interaction of PGRMC1 with HK2.}

We used phosphomimetics to determine if differential phosphorylation at S57 in HEK293 and HepG2 cells (Fig 4F-H) is responsible for the cell-type-specific interaction of PGRMC1 with HK2 (Fig 5 \& 6). The S57 residue overlaps a potential Src homology 3 (SH3) domain on PGRMC1 [62] and the SH3 domains have been identified as protein modules that recognize proline-rich sequences [63]. The existence of a multimeric protein complex involving HK2 and Src tyrosine kinase has been reported[64]. Therefore, sitedirected mutagenesis was used to generate phosphonull PGRMC1(S57A) and phosphomimic PGRMC1(S57D/E) mutants (Fig S7A). DNA sequencing confirmed the desired mutations in the plasmids expressing Flag-tagged PGRMC1 (Fig S7B). Next, we transiently transfected Flag-PGRMC1 mutants in HEK293 cells and the anti-Flag immunoprecipitate was subjected to immunoblotting using HK-specific antibodies. Immunoblotting revealed the expression of Flag-PGRMC1 mutants in HEK293 cells (Fig S7C), while Oriole-staining and immunoblotting revealed Flag-PGRMC1 mutants could be immunoprecipitated (Fig S7D-E). Immunoblotting revealed the presence of both HK1 and HK2 in the mutant FlagPGRMC1(S57A/D/E) immunoprecipitates indicating S57 phosphorylation is not involved in the cell-typespecific interaction of PGRMC1 and HK2 (Fig S7F). However, plot profiling revealed the relative abundance of an unknown p75 kDa interacting protein (Fig S7D, blue arrow) which is relatively more abundant in the PGRMC1 phosphonull mutant (S57A) immunoprecipitate compared to the wild-type (S57) or phosphomimic mutants (S57D/E). These observations suggest that the phosphorylation state of S47 may influence the interaction of PGRMC1 with other proteins but not HKs (Fig S7G). Identification of the p75 interacting protein will be the subject of future studies.

\section{Distribution of HKs in different subcellular compartments.}

The subcellular distribution of HK1 and HK2 has been proposed to regulate metabolic preference by cells[43]. A dual role has been proposed for HK2 based on its subcellular location, which involves channeling glucose-6-phosphate (G6P) into glycogen and PPP when localized in the cytoplasm. In contrast, shuttling G6P to glycolysis and OXPHOS occurs when HK2 is bound to mitochondria [65]. Therefore, P4-induced subcellular localization of HK1 and HK2 in HEK293 and HepG2 cells was examined. Immunoblotting revealed that both HK1 and HK2 are predominantly associated with the ER/mitochondrial fraction (HK1: 72.5\% vs HK2: 64.9\%) compared to the cytosolic (HK1: $11.4 \%$ vs HK2: $22.2 \%$ ) and nuclear (HK1: 16.1\% vs HK2: 12.9\%) fractions in HEK293 cells (Fig 7A-F). In contrast to HEK293 cells, HK1 and HK2 are distributed across all cell compartments, including the cytosol (HK1: 9.9\% 
vs HK2: 23.1\%), F1 ER/mitochondrial fraction (HK1: 33.4\% vs HK2: 24.6\%), F2 ER/mitochondrial fraction (HK1: 22.5\% vs HK2: 28\%) and the nucleus (HK1: 34.2\% vs HK2: 24.3\%) in HepG2 cells (Fig 7G-L). In the cytosol of both HEK293 and HepG2 cells, the relative amount of HK1 was significantly higher than HK2 (Fig 7FL). The HK1 level was also significantly higher than HK2 in the nucleus of HepG2 cells, whereas both proteins remained at the same level in the nucleus of HEK293 cells (Fig 7L). Likewise, the ER/mitochondrial fractions from HEK293 and HepG2 (F1 fraction) cells contained significantly higher HK1 compared to HK2 (Fig 7FI). Thus, the overall distribution of HK1 and HK2 is similar in both cell types, with HK1 predominantly present in the ER/mitochondria/nucleus versus HK2 which is predominantly found in the cytosol.

\section{P4 treatment causes cell type-specific differential distribution of HKs in subcellular compartments.}

Immunoblotting was used to compare the HK content in subcellular fractions from the vehicle and P4treated HEK293 cells. This analysis revealed that P4 treatment caused a significant reduction of both HK1 and HK2 in the ER/mitochondrial fraction compared to the vehicle-treated cells (Fig 8A-D). In contrast to HEK293 cells, the HK1 and HK2 levels were found significantly increased in the $\mathrm{ER} /$ mitochondrial fractions (F1) of P4-treated HepG2 cells compared to vehicle-treated cells (Fig 8E-H). In both HEK293 and HepG2 cells, P4-treatment significantly increased translocation of HK1 to the nucleus compared to the vehicle-treated cells (Fig 8IK \& MO). In contrast to HK1, the HK2 level was significantly decreased in the HEK293 nuclear fraction (Fig 8JL), but increased in the HepG2 nuclear fraction compared to vehicle-treated cells (Fig 8LP). Overall, these results indicate a considerable difference in the effect of P4 on subcellular translocation of HK1 and HK2 proteins in HEK293 and HepG2 cells. This differential action may account for the metabolic differences induced by $\mathrm{P} 4$.

\section{Discussion}

This study provides evidence that the ability of P4 to modulate glycolysis is cell-type-specific. P4 caused a rapid increase in glycolysis in HEK293 cells, whereas it decreased glycolysis in HepG2 cells. These two extremes of P4-induced glucose utilization were found associated with differential PGRMC1 PTMs, and proteasomal degradation of PGRMC1 and cell-type-specific interaction with HKs. The identification of HKs as an interacting partner of PGRMC1 is novel. Also, PGRMC1-mediated regulation of ER and MAM structures in HepG2 cells is a novel finding that provides a direct link to potential metabolic regulatory mechanisms. Furthermore, P4 treatment of HEK293 and HepG2 cells caused a cell-type-specific differential translocation of HK1/2 to the ER and mitochondrial compartments as well as the nucleus which may account for the metabolic responses by these two cell types to P4.

PGRMC1 was found differentially phosphorylated in HEK293 and HepG2 cells under basal conditions (Fig 4\&5). P-PGRMC1 (T-178, Y180, and S181) residues were detected in HepG2 cells, which is in contrast to previously identified P-PGRMC1 (S57 \& S181) residues in HEK293 cells[3]. The significance of PGRMC1 phosphorylation is not completely known. Differential phosphorylation (charged fraction) of PGRMC1 in estrogen receptor-positive (ER) and ER-negative breast cancers was reported byNeubauer et 
al. [66]. Breast tumors are sex hormone-sensitive and $₫ 60-70 \%$ of all breast cancers are either estrogen receptor (ER)-positive or P4-receptor (PR)-positive [67, 68]. Clinically, hormone-receptor-positive cancers tend to grow more slowly and have favorable clinical outcomes than hormone-receptor-negative tumors[69]. The exact mechanism for such a difference in terms of disease outcome is not completely understood. The most frequently observed PGRMC1 phosphorylation sites listed in the PhosphositePlus database are S57 and S181 followed by Y113, Y180, and T178 [3, 55, 61]. The conserved S57 and S181 residues overlap predicted Src homology domain binding motifs and also match to a theoretically predicted site for casein kinase 2 (CK2) activity[62]. Previously, reduced levels of P-PGRMC1(Ser) were found associated with the P4-induced Warburg effect in HEK293 cells[3]. Interestingly, in HepG2 cells, P4induced reduction in glycolysis is not associated with a reduction in P-PGRMC1(Ser) levels. Also, the absence of P-PGRMC1(S57) in HepG2 cells under basal condition indicates a correlation with PGRMC1 phosphorylation state and P4-induced metabolic reprogramming. Neubauer et. al. showed that stable transfection of S57A and S180A phosphonull mutants in MCF7 breast cancer cells protected cells from peroxide-induced cell death[66]. In a recent study, progestin treatment of MCF7 and T47D breast cancer cell lines increased P-PGRMC1 (S181) levels which were subsequently reduced by treatment with a CK2 inhibitor[70]. Thus, a link between PGRMC1 phosphorylation involving S57 and S180 residues and breast cancer pathology has been established, however, the mechanistic role of these two sites in disease pathophysiology is still not well established. Another important aspect of PGRMC1 S57 and S180 phosphorylation state is that it may affect potential interacting protein binding to the putative $\mathrm{SH}$ domain. Based on that understanding, we used S57 phosphomimic (S57D/E) and phosphonull (S57A) mutants to study the interaction of PGRMC1 with HK2 (Fig S7). Interestingly, phosphomimetics revealed that S57 phosphorylation status is not responsible for the cell type-specific interaction of PGRMC1 and HK2, however, the Oriole-stained immunoprecipitated proteins revealed an unknown $75 \mathrm{kDa}$ interacting protein that exhibited differential interaction with S57A compared to the native S57 and S57D/E PGRMC1 in HEK293 cells (Fig S7G).

PGRMC1 is a $25 \mathrm{kDa}$ protein. Complex PTMs of PGRMC1 including SUMOylation and ubiquitination were found associated with the appearance of higher molecular mass forms $(70-100 \mathrm{kDa})$ of the protein in the $\mathrm{ER} /$ mitochondria and nuclear fractions in HEK293 cells [3]. SUMO and ubiquitin have a molecular weight of $\sim 8-10 \mathrm{kDa}[71,72]$, therefore, it was suggested that the appearance of high molecular weight PGRMC1 was due to polyubiquitination and/or SUMOylation [3]. Multiple ubiquitination cycles add polyubiquitin chain on a protein which acts as a signal for proteasomal degradation [71]. A previous study demonstrated that P4-induced glycolysis and rapid proteasomal degradation of p70 PGRMC1 in HEK293 cells is associated with altered levels of PGRMC1 SUMOylation and ubiquitination. Therefore, the absence of any alteration in the PGRMC1 SUMOylation and ubiquitination is in agreement with the resistance of PGRMC1 to P4-induced proteasomal degradation and reduced glycolytic activity in HepG2 cells. Furthermore, phosphorylation cross-talks with SUMOylation and ubiquitination to determine the fate of a protein [73]. Interestingly, the P-PGRMC1 (Ser) level in P4-treated HepG2 cells was unaltered, which is in contrast to the HEK293 cells where P4-induced reduction of P-PGRMC1 (Ser) level was demonstrated. The difference in the PTMs of PGRMC1 under basal condition may also be responsible for the cell-type- 
specific differential half-life of the protein (Fig S3). Thus, it is evident that phosphorylation and SUMOylation/ubiquitination of PGRMC1 are linked to proteasomal degradation in the context of P4induced cell type-specific metabolic reprogramming, however, the exact mechanism needs to be elucidated in the future.

This study highlighted cell-type-specific differential interaction of HK1/2 and PGRMC1 (Fig 7), as well as a differential distribution of HK1/2 in various subcellular organelles following P4-stimulation (Fig 8). Both processes may contribute to $\mathrm{P} 4$-induced cell type-specific metabolic reprogramming. Mammalian hexokinases are allosteric enzymes catalyzing ATP-dependent phosphorylation of glucose to G6P [74, 75]. Despite sequence similarities, the kinetic features, regulatory properties, intracellular distribution, and expression pattern of HKs differ significantly [44]. For example, the HK-mediated reaction product G6P inhibits both HK1 and HK2 at micromolar levels; however, inorganic phosphate (Pi) only relieves G6P inhibition of HK1 [75]. Another important fundamental feature that distinguishes these isoforms is the unique capacity of HK1 and HK2 to directly interact with mitochondria, both physically and functionally [44]. Wilson et al. proposed that mitochondria-bound HK1 couples cytosolic glycolysis to intramitochondrial OXPHOS, whereas HK2 serves a primarily anabolic function to provide G6P for glycogen or lipid synthesis via the PPP (Fig S6). Therefore, P4-induced translocation of HK1 and 2 away from the ER/mitochondria may suppress OXPHOS activity (respiration) but increase glycolysis in HEK293 cells. On the other hand, increased translocation of HK1 and HK2 to the ER/mitochondria in P4-treated HepG2 cells may lead to a suppression of both glycolysis and respiration. It has been suggested that the primary effect of P4 on carbohydrate metabolism is the promotion of glycogen storage in the liver [30]. P4 has been shown to induce glycogen synthesis in a dose-dependent manner in primary human endometrial cells which express a high level of PGRMC1[76]. In this context, it is important to note that in our study the rate of glycolysis was measured in terms of ECAR (due to lactate secretion) which does not reflect the true status of glycolysis in the cell. For example, if G6P production due to increased glycolytic activity is channeled to glycogen synthesis or the PPP, then ECAR values will not change. As a result, the Seahorse flux analyzer will not be able to detect an increase in glycolysis. Therefore, increased channeling of G6P to glycogen synthesis or the PPP in response to P4 cannot be excluded in HepG2 cells. However, in a contrasting study, $\mathrm{HK} 1 / 2$ overexpression in $\mathrm{CHO}$ cells revealed that $\mathrm{HK} 1$ exclusively promotes glycolysis, whereas HK2 has a more complex role, promoting glycolysis when bound to the mitochondria and glycogen synthesis when located in the cytosol[77]. Thus, the exact mechanism of P4-induced cell typespecific differential translocation of HKs and metabolic reprogramming cannot be deciphered in this study and more in-depth studies in the future will be necessary to shed light on this important aspect of metabolic regulation.

The loss of PGRMC1 altered ER and mitochondrial organization in HepG2 cells. The ER is a complex dynamic organelle, primarily involved in protein and lipid synthesis as well as secretion, calcium homeostasis, and interactions with other organelles[78]. The structural variations of ER in different celltypes are a reflection of functional heterogeneity [79]. Therefore, the differences in ER structure in HepG2 and HEK293 cells were anticipated and this difference may account for the P4-induced metabolic difference in these cell-types to some extent. The ER tubules in PGRMC1-deficient HepG2 cells were 
fragmented compared to the wild-type suggesting PGRMC1 has a role in the maintenance of ER structural integrity (Fig 4). The continuity of the ER lumen is vital for this function and is required for the efficient diffusion and equilibration of ions[80]. The integrity of the ER and MAM is also required for hormonal signaling and glucose homeostasis [81] and its alteration is associated with the pathogenesis of metabolic diseases[82]. The MAM interface acts as a nutrient-regulated hub that adapts mitochondrial metabolism to the nutritional state of the cell [83]. In nutrient-limited conditions, the ER-mitochondria interaction doubles in length in liver cells [84], whereas high glucose levels disrupt MAM integrity in the liver [59]. These findings suggest that the liver could adapt to metabolic transition through a MAMdependent mechanism. In mammalian MAM, the ER and mitochondria are physically tethered to each other by the inositol 1,4,5-trisphosphate (IP3) receptor $\left(\mathrm{IP}_{3} \mathrm{R} / \mathrm{ITPR}\right)$-glucose-regulated protein 75 (Grp75/HSPA9)-VDAC trimeric complex (Fig S6) [85]. VDAC interacts with adenine nucleotide translocase (ANT), an inner membrane protein (Fig S6) [86]. The resultant VDAC-ANT-mitochondrial creatine kinase (mCK) complex regulates the exchange of ATP and ADP between the mitochondria and cytosol [87]. Both HK1 and HK2 directly interact with and promote the 'open' configuration of VDAC that permits anionic metabolite exchange across the outer mitochondrial membrane (OMM)[44, 88]. Functional coupling between glycolysis and OXPHOS is mediated by ADP-ATP exchange through the VDAC-ANT complex (Fig S5)[44]. Therefore, the association of PGRMC1 with HKs, P4-induced alteration of the subcellular distribution of HKs and metabolic reprogramming, and abnormal MAM in PGRMC1-deficient cells all point to a MAM-based metabolic transition switch controlled by P4-PGRMc1 signaling.

\section{Conclusions}

Glucose is the preferred fuel for ATP production by most cells. The fate of pyruvate, a product of glycolysis, depends upon whether oxygen is present. For example, when oxygen levels are low, skeletal muscle cells rely on glycolysis to meet their intense energy requirement and generate lactic acid as a byproduct [89]. In the presence of oxygen, the pyruvate is converted into acetyl CoA and subsequently enters the mitochondria to generate 36 molecules of ATP through the tricarboxylic acid (TCA) cycle and oxidative phosphorylation (OXPHOS). Thus, it is conceivable that burning glucose through glycolysis, TCA cycle, and OXPHOS pathways is a profitable metabolic preference for a cell. However, in tumors and other proliferating or developing cells, the rate of glucose uptake dramatically increases and the metabolic preference shifts to the production of lactate, even in the presence of oxygen and fully functioning mitochondria [90]. This process, known as the Warburg effect or aerobic glycolysis [91], is favored by tumor cells because the interior cells in a tumor mass may experience fluctuations in oxygen tension that in turn limit nutrient oxidation; therefore, shifting the metabolic preference to aerobic glycolysis gives an advantage for tumor cell survival [90]. Besides, increased glucose utilization generates high amounts of lactate, which creates an acidic environment and facilitates tumor invasion [90]. The metabolic preference for aerobic glycolysis over OXPHOS also depends on other factors, for example, hormones. Decoding the mechanism of metabolic reprogramming is vital to our understanding of the diseases associated with metabolic dysfunction. Thus, our discovery of a P4-PGRMC1 signaling 
mediated cell type-specific metabolic regulation pathway is an important step towards unraveling potentially new mechanisms underlying the hormone-responsive tumor phenotype.

\section{List Of Abbreviations}

ECAR: extracellular acidification rate

OCR: oxygen consumption rate

OMM: outer mitochondrial membrane

IEF: Isoelectric focusing.

P4: progesterone

kDa: kilo daltons

OXPHOS: oxidative phosphorylation

TCA: Tricarboxylic acid cycle

\section{Declarations}

Ethics approval and consent to participate: Not applicable

Consent for publication: Not applicable

Availability of data and materials: The datasets used and/or analyzed during the current study are available from the corresponding author on reasonable request.

Competing interests: The authors declare that they have no competing interests

Funding: Alzo Bioscience Inc., USA provided some reagent support and St. Boniface Hospital Albrechtsen Research Centre provided additional funding and infrastructure support.

Authors' contributions: Mohammad Golam Sabbir: Conceptualization, Investigation, Formal analysis, Visualization, Writing - original draft. Carla G. Taylor: Writing - review \& editing, Resources. Peter Zahradka: Writing - review \& editing, Resources. All authors read and approved the final manuscript.

Acknowledgements:We acknowledge Alzo Bioscience Inc., USA for reagent support; St. Boniface Hospital Albrechtsen Research Centre for additional funding and infrastructure support; Jennifer Chung, Santa Cruz Biotechnology Inc. for providing numerous mouse monoclonal antibodies; Andrew Pobre, Medical Laboratory Technologist, Diagnostic Services Manitoba, for excellent electron microscope sample preparation; Laura Curtis, research specialist, Shared Health Manitoba, for access to the electron 
microscope facility; Ying Lao, Manitoba Centre for Proteomics and Systems Biology, for mass spectrometry data acquisition service.

\section{References}

1. Ryu, C.S., K. Klein, and U.M. Zanger, Membrane Associated Progesterone Receptors: Promiscuous Proteins with Pleiotropic Functions - Focus on Interactions with Cytochromes P450. Front Pharmacol, 2017. 8: p. 159.

2. Fagerberg, L., et al., Analysis of the human tissue-specific expression by genome-wide integration of transcriptomics and antibody-based proteomics. Mol Cell Proteomics, 2014. 13(2): p. 397-406.

3. Sabbir, M.G., Progesterone induced Warburg effect in HEK293 cells is associated with posttranslational modifications and proteasomal degradation of progesterone receptor membrane component 1. J Steroid Biochem Mol Biol, 2019. 191: p. 105376.

4. Mir, S.U., et al., Progesterone receptor membrane component 1/Sigma-2 receptor associates with MAP1LC3B and promotes autophagy. Autophagy, 2013. 9(10): p. 1566-78.

5. Peluso, J.J., et al., Progesterone receptor membrane component-1 (PGRMC1) and PGRMC-2 interact to suppress entry into the cell cycle in spontaneously immortalized rat granulosa cells. Biol Reprod, 2014. 91(5): p. 104.

6. Peluso, J.J., et al., Regulation of ovarian cancer cell viability and sensitivity to cisplatin by progesterone receptor membrane component-1. J Clin Endocrinol Metab, 2008. 93(5): p. 1592-9.

7. Peluso, J.J., et al., Progesterone receptor membrane component 1 and 2 regulate granulosa cell mitosis and survival through a NFKappaB-dependent mechanismdagger. Biol Reprod, 2019. 100(6): p. 15711580.

8. Clark, N.C., et al., Progesterone receptor membrane component 1 promotes survival of human breast cancer cells and the growth of xenograft tumors. Cancer Biol Ther, 2016. 17(3): p. 262-71.

9. Sueldo, C., X. Liu, and J.J. Peluso, Progestin and AdipoQ Receptor 7, Progesterone Membrane Receptor Component 1 (PGRMC1), and PGRMC2 and Their Role in Regulating Progesterone's Ability to Suppress Human Granulosa/Luteal Cells from Entering into the Cell Cycle. Biol Reprod, 2015. 93(3): p. 63.

10. Hughes, A.L., et al., Dap1/PGRMC1 binds and regulates cytochrome P450 enzymes. Cell Metab, 2007. 5(2): p. 143-9.

11. Ahmed, I.S., C. Chamberlain, and R.J. Craven, S2R(Pgrmc1): the cytochrome-related sigma-2 receptor that regulates lipid and drug metabolism and hormone signaling. Expert Opin Drug Metab Toxicol, 2012. 8(3): p. 361-70. 
12. Li, X., et al., Progesterone receptor membrane component-1 regulates hepcidin biosynthesis. J Clin Invest, 2016. 126(1): p. 389-401.

13. Piel, R.B., 3rd, et al., A Novel Role for Progesterone Receptor Membrane Component 1 (PGRMC1): A Partner and Regulator of Ferrochelatase. Biochemistry, 2016. 55(37): p. 5204-17.

14. Mansouri, M.R., et al., Alterations in the expression, structure and function of progesterone receptor membrane component-1 (PGRMC1) in premature ovarian failure. Hum Mol Genet, 2008. 17(23): p. 377683.

15. Guo, M., et al., Progesterone Receptor Membrane Component 1 Mediates Progesterone-Induced Suppression of Oocyte Meiotic Prophase I and Primordial Folliculogenesis. Sci Rep, 2016. 6: p. 36869.

16. Luciano, A.M., et al., Progesterone receptor membrane component 1 expression and putative function in bovine oocyte maturation, fertilization, and early embryonic development. Reproduction, 2010. 140(5): p. 663-72.

17. Izzo, N.J., et al., Alzheimer's therapeutics targeting amyloid beta 1-42 oligomers II: Sigma-2/PGRMC1 receptors mediate Abeta 42 oligomer binding and synaptotoxicity. PLoS One, 2014. 9(11): p. e111899.

18. Kim, J.Y., et al., Progesterone Receptor Membrane Component 1 suppresses the $p 53$ and Wnt/betacatenin pathways to promote human pluripotent stem cell self-renewal. Sci Rep, 2018. 8(1): p. 3048.

19. Shih, C.C., et al., Role of PGRMC1 in cell physiology of cervical cancer. Life Sci, 2019. 231: p. 116541.

20. Neubauer, $\mathrm{H}_{\text {., }}$ et al., Possible role of PGRMC1 in breast cancer development. Climacteric, 2013. 16(5): p. 509-13.

21. Mir, S.U., et al., Elevated progesterone receptor membrane component 1/sigma-2 receptor levels in lung tumors and plasma from lung cancer patients. Int J Cancer, 2012. 131(2): p. E1-9.

22. Peluso, J.J., Progesterone signaling mediated through progesterone receptor membrane component-1 in ovarian cells with special emphasis on ovarian cancer. Steroids, 2011. 76(9): p. 903-9.

23. Willibald, M., et al., High Level of Progesteron Receptor Membrane Component 1 (PGRMC 1) in Tissue of Breast Cancer Patients is Associated with Worse Response to Anthracycline-Based Neoadjuvant Therapy. Horm Metab Res, 2017. 49(8): p. 595-603.

24. Lange, C.A. and D. Yee, Progesterone and breast cancer. Womens Health (Lond), 2008. 4(2): p. 151-62.

25. Trabert, B., et al., Progesterone and Breast Cancer. Endocr Rev, 2020. 41(2).

26. Kabe, Y., et al., Haem-dependent dimerization of PGRMC1/Sigma-2 receptor facilitates cancer proliferation and chemoresistance. Nat Commun, 2016. 7: p. 11030. 
27. Peluso, J.J., J. Romak, and X. Liu, Progesterone Receptor Membrane Component-1 (PGRMC1) Is the Mediator of Progesterone's Antiapoptotic Action in Spontaneously Immortalized Granulosa Cells As Revealed by PGRMC1 Small Interfering Ribonucleic Acid Treatment and Functional Analysis of PGRMC1 Mutations. Endocrinology, 2008. 149(2): p. 534-543.

28. Schumacher, M., et al., Progesterone synthesis in the nervous system: implications for myelination and myelin repair. Front Neurosci, 2012. 6: p. 10.

29. Acconcia, F. and M. Marino, Principles of Endocrinology and Hormone Action. Steroid Hormones: Synthesis, Secretion, and Transport, ed. A. Lenzi. Switzerland: Springer.

30. Kalkhoff, R.K., Metabolic effects of progesterone. Am J Obstet Gynecol, 1982. 142(6 Pt 2): p. 735-8.

31. Bowman, K. and J. Rose, Estradiol stimulates glycogen synthesis whereas progesterone promotes glycogen catabolism in the uterus of the American mink (Neovison vison). Anim Sci J, 2017. 88(1): p. 4554.

32. Gras, F., et al., Progesterone impairs cell respiration and suppresses a compensatory increase in glucose transport in isolated rat skeletal muscle: a non-genomic mechanism contributing to metabolic adaptation to late pregnancy? Diabetologia, 2007. 50(12): p. 2544-52.

33. Epstein, T., R.A. Gatenby, and J.S. Brown, The Warburg effect as an adaptation of cancer cells to rapid fluctuations in energy demand. PLoS One, 2017. 12(9): p. e0185085.

34. Diep, C.H., et al., Progesterone action in breast, uterine, and ovarian cancers. J Mol Endocrinol, 2015. 54(2): p. R31-53.

35. Kasubuchi, M., et al., Membrane progesterone receptor beta (mPRbeta/Paqr8) promotes progesteronedependent neurite outgrowth in PC12 neuronal cells via non-G protein-coupled receptor (GPCR) signaling. Sci Rep, 2017. 7(1): p. 5168.

36. Garg, D., et al., Progesterone-Mediated Non-Classical Signaling. Trends Endocrinol Metab, 2017. 28(9): p. 656-668.

37. Falkenstein, E., et al., Localization of a putative progesterone membrane binding protein in porcine hepatocytes. Cell Mol Biol (Noisy-le-grand), 1998. 44(4): p. 571-8.

38. Peluso, J.J., V. Lodde, and X. Liu, Progesterone regulation of progesterone receptor membrane component 1 (PGRMC1) sumoylation and transcriptional activity in spontaneously immortalized granulosa cells. Endocrinology, 2012. 153(8): p. 3929-39.

39. Su, C., et al., Progesterone increases the release of brain-derived neurotrophic factor from glia via progesterone receptor membrane component 1 (Pgrmc1)-dependent ERK5 signaling. Endocrinology, 2012. 153(9): p. 4389-400. 
40. Peluso, J.J., et al., Expression and function of PAIRBP1 within gonadotropin-primed immature rat ovaries: PAIRBP1 regulation of granulosa and luteal cell viability. Biol Reprod, 2005. 73(2): p. 261-70.

41. Jin, L. and Y. Zhou, Crucial role of the pentose phosphate pathway in malignant tumors. Oncol Lett, 2019. 17(5): p. 4213-4221.

42. Katzen, H.M. and R.T. Schimke, Multiple forms of hexokinase in the rat: tissue distribution, age dependency, and properties. Proc Natl Acad Sci U S A, 1965. 54(4): p. 1218-25.

43. Wilson, J.E., Isozymes of mammalian hexokinase: structure, subcellular localization and metabolic function. J Exp Biol, 2003. 206(Pt 12): p. 2049-57.

44. Robey, R.B. and N. Hay, Mitochondrial hexokinases, novel mediators of the antiapoptotic effects of growth factors and Akt. Oncogene, 2006. 25(34): p. 4683-96.

45. Hiller, S., et al., Solution structure of the integral human membrane protein VDAC-1 in detergent micelles. Science, 2008. 321(5893): p. 1206-10.

46. Uhlen, M., et al., A human protein atlas for normal and cancer tissues based on antibody proteomics. Mol Cell Proteomics, 2005. 4(12): p. 1920-32.

47. Darlington, G.J., J.H. Kelly, and G.J. Buffone, Growth and hepatospecific gene expression of human hepatoma cells in a defined medium. In Vitro Cell Dev Biol, 1987. 23(5): p. 349-54.

48. Sabbir, M.G., C.G. Taylor, and P. Zahradka, Hypomorphic CAMKK2 in EA.hy926 endothelial cells causes abnormal transferrin trafficking, iron homeostasis and glucose metabolism. Biochim Biophys Acta Mol Cell Res, 2020: p. 118763.

49. Hartig, S.M., Basic image analysis and manipulation in ImageJ. Curr Protoc Mol Biol, 2013. Chapter 14: p. Unit14 15.

50. Li, A., et al., Use of nitrocellulose membranes as a scaffold in cell culture. Cytotechnology, 2013. 65(1): p. 71-81.

51. Dunn, O.J., Multiple Comparisons Using Rank Sums. Technometrics, 1964. 6(3): p. 241-252.

52. Siegel, S., ed. Nonparametric statistics for the behavioral sciences. McGraw-Hill series in psychology. 1956, McGraw-Hill: New York. pages 312.

53. Muller-Taubenberger, A., et al., Calreticulin and calnexin in the endoplasmic reticulum are important for phagocytosis. EMBO J, 2001. 20(23): p. 6772-82.

54. Mihara, K. and R. Sato, Molecular cloning and sequencing of cDNA for yeast porin, an outer mitochondrial membrane protein: a search for targeting signal in the primary structure. EMBO J, 1985. 4(3): p. 769-74. 
55. Hornbeck, P.V., et al., PhosphoSitePlus: a comprehensive resource for investigating the structure and function of experimentally determined post-translational modifications in man and mouse. Nucleic Acids Res, 2012. 40(Database issue): p. D261-70.

56. Tan, B., et al., The profiles of mitochondrial respiration and glycolysis using extracellular flux analysis in porcine enterocyte IPEC-J2. Anim Nutr, 2015. 1(3): p. 239-243.

57. Eden, E., et al., Proteome half-life dynamics in living human cells. Science, 2011. 331(6018): p. 764-8.

58. Zhou, P., Determining protein half-lives. Methods Mol Biol, 2004. 284: p. 67-77.

59. Theurey, P., et al., Mitochondria-associated endoplasmic reticulum membranes allow adaptation of mitochondrial metabolism to glucose availability in the liver. J Mol Cell Biol, 2016. 8(2): p. 129-43.

60. Stacchiotti, A., et al., Perspective: Mitochondria-ER Contacts in Metabolic Cellular Stress Assessed by Microscopy. Cells, 2018. 8(1).

61. Hornbeck, P.V., et al., PhosphoSitePlus, 2014: mutations, PTMs and recalibrations. Nucleic Acids Res, 2015. 43(Database issue): p. D512-20.

62. Cahill, M.A., et al., PGRMC1 regulation by phosphorylation: potential new insights in controlling biological activity. Oncotarget, 2016. 7(32): p. 50822-50827.

63. Kaneko, T., L. Li, and S.S. Li, The SH3 domain-a family of versatile peptide- and protein-recognition module. Front Biosci, 2008. 13: p. 4938-52.

64. Pantic, B., et al., Myotonic dystrophy protein kinase (DMPK) prevents ROS-induced cell death by assembling a hexokinase II-Src complex on the mitochondrial surface. Cell Death \& Disease, 2013. 4(10): p. e858-e858.

65. Jurczak, M.J., et al., The role of protein translocation in the regulation of glycogen metabolism. J Cell Biochem, 2008. 104(2): p. 435-43.

66. Neubauer, $\mathrm{H}$., et al., Breast cancer proteomics reveals correlation between estrogen receptor status and differential phosphorylation of PGRMC1. Breast Cancer Res, 2008. 10(5): p. R85.

67. Harbeck, N., et al., Breast cancer. Nat Rev Dis Primers, 2019. 5(1): p. 66.

68. Shah, R., K. Rosso, and S.D. Nathanson, Pathogenesis, prevention, diagnosis and treatment of breast cancer. World J Clin Oncol, 2014. 5(3): p. 283-98.

69. Nasrazadani, A., et al., Precision Medicine in Hormone Receptor-Positive Breast Cancer. Front Oncol, 2018. 8: p. 144. 
70. Willibald, M., et al., Progesterone receptor membrane component 1 is phosphorylated upon progestin treatment in breast cancer cells. Oncotarget, 2017. 8(42): p. 72480-72493.

71. Xu, G. and S.R. Jaffrey, Proteomic identification of protein ubiquitination events. Biotechnol Genet Eng Rev, 2013. 29: p. 73-109.

72. Santos, A.L. and A.B. Lindner, Protein Posttranslational Modifications: Roles in Aging and Age-Related Disease. Oxid Med Cell Longev, 2017. 2017: p. 5716409.

73. Hunter, T., The age of crosstalk: phosphorylation, ubiquitination, and beyond. Mol Cell, 2007. 28(5): p. 730-8.

74. Magnani, M., et al., A recombinant human 'mini'-hexokinase is catalytically active and regulated by hexose 6-phosphates. Biochem J, 1992. 285 ( Pt 1): p. 193-9.

75. Aleshin, A.E., et al., The mechanism of regulation of hexokinase: new insights from the crystal structure of recombinant human brain hexokinase complexed with glucose and glucose-6-phosphate. Structure, 1998. 6(1): p. 39-50.

76. Ishihara, S., Y. Taketani, and M. Mizuno, Stimulatory action of progesterone on the synthesis of glycogen in primary cell culture of human endometrium. Asia Oceania J Obstet Gynaecol, 1988. 14(1): p. 117-22.

77. John, S., J.N. Weiss, and B. Ribalet, Subcellular localization of hexokinases I and II directs the metabolic fate of glucose. PLoS One, 2011. 6(3): p. e17674.

78. Schwarz, D.S. and M.D. Blower, The endoplasmic reticulum: structure, function and response to cellular signaling. Cell Mol Life Sci, 2016. 73(1): p. 79-94.

79. Baumann, O. and B. Walz, Endoplasmic reticulum of animal cells and its organization into structural and functional domains. Int Rev Cytol, 2001. 205: p. 149-214.

80. Kucharz, K., T. Wieloch, and H. Toresson, Rapid fragmentation of the endoplasmic reticulum in cortical neurons of the mouse brain in situ following cardiac arrest. J Cereb Blood Flow Metab, 2011. 31(8): p. 1663-7.

81. Rieusset, J., The role of endoplasmic reticulum-mitochondria contact sites in the control of glucose homeostasis: an update. Cell Death Dis, 2018. 9(3): p. 388.

82. Tubbs, E., et al., Mitochondria-associated endoplasmic reticulum membrane (MAM) integrity is required for insulin signaling and is implicated in hepatic insulin resistance. Diabetes, 2014. 63(10): p. 3279-94. 
83. Theurey, P. and J. Rieusset, Mitochondria-Associated Membranes Response to Nutrient Availability and Role in Metabolic Diseases. Trends Endocrinol Metab, 2017. 28(1): p. 32-45.

84. Sood, A., et al., A Mitofusin-2-dependent inactivating cleavage of Opa 1 links changes in mitochondria cristae and ER contacts in the postprandial liver. Proc Natl Acad Sci U S A, 2014. 111(45): p. 16017-22.

85. Szabadkai, G., et al., Chaperone-mediated coupling of endoplasmic reticulum and mitochondrial Ca2+ channels. J Cell Biol, 2006. 175(6): p. 901-11.

86. Allouche, M., et al., ANT-VDAC1 interaction is direct and depends on ANT isoform conformation in vitro. Biochem Biophys Res Commun, 2012. 429(1-2): p. 12-7.

87. Camara, A.K.S., et al., Mitochondrial VDAC1: A Key Gatekeeper as Potential Therapeutic Target. Front Physiol, 2017. 8: p. 460.

88. Pastorino, J.G. and J.B. Hoek, Regulation of hexokinase binding to VDAC. J Bioenerg Biomembr, 2008. 40(3): p. 171-82.

89. Rogatzki, M.J., et al., Lactate is always the end product of glycolysis. Front Neurosci, 2015. 9: p. 22.

90. Liberti, M.V. and J.W. Locasale, The Warburg Effect: How Does it Benefit Cancer Cells? Trends Biochem Sci, 2016. 41(3): p. 211-218.

91. Warburg, O., F. Wind, and E. Negelein, The Metabolism of Tumors in the Body. J Gen Physiol, 1927. 8(6): p. 519-30.

92. Beavis, R.C., Using the global proteome machine for protein identification. Methods Mol Biol, 2006. 328: p. 217-28.

93. Betz, C., et al., Feature Article: mTOR complex 2-Akt signaling at mitochondria-associated endoplasmic reticulum membranes (MAM) regulates mitochondrial physiology. Proc Natl Acad Sci U S A, 2013. 110(31): p. 12526-34.

94. Szado, T., et al., Phosphorylation of inositol 1,4,5-trisphosphate receptors by protein kinase B/Akt inhibits Ca2+ release and apoptosis. Proc Natl Acad Sci U S A, 2008. 105(7): p. 2427-32.

\section{Tables}

\section{Table-1: List of reagents.}




\begin{tabular}{|llll|}
\hline Antibodies & & & \\
\hline Name & Species/Type & Source & Catalogue \\
\hline Anti-PGRMC1 & Mouse monoclonal & SCBT & SC-363015 \\
\hline Flag & Rabbit polyclonal & Biorybt & ORB167451 \\
\hline Flag & Mouse monoclonal & Sigma & F3165 \\
\hline Calnexin (CANX) & Mouse monoclonal & SCBT & Sc-46669 \\
\hline VDAC1 & Mouse monoclonal & SCBT & Sc-390996 \\
\hline aTubulin (TUB1A) & Mouse monoclonal & SCBT & Sc-8035 \\
\hline HK1 & Rabbit Polyclonal & CST & $2804 S$ \\
\hline HK1 & Mouse monoclonal & SCBT & Sc-80978 \\
\hline HK2 & Rabbit monoclonal & CST & $2867 S$ \\
\hline HK2 & Mouse monoclonal & SCBT & SC374091 \\
\hline
\end{tabular}




\begin{tabular}{|ll|}
\hline CRISPR/Cas9 constructs & \\
\hline CRISPR/Cas9-PGRMC1 & sc-401945 (SCBT) \\
\hline HDR- cassette & sc-401945-HDR(SCBT) \\
\hline Primers/gRNAs & Sequence (5'-3') \\
\hline PGRMC1 gRNA-A & TCCAGTCAAGTATCATCACG \\
\hline PGRMC1 gRNA-B & GGCCGCAAATTCTACGGGCC \\
\hline PGRMC1 gRNA-C & CTTCCAGCAAAGACCCCATA \\
\hline Full length ORF amplification primers \\
\hline PGRMC1 Forward & GACCCAAGCGATCTGGAGAG \\
\hline PGRMC1 Reverse & GACAGAGTGGACTGTTACAAATG \\
\hline PGRMC2 Forward & GGGACGTGAAGCTAGGCA \\
\hline PGRMC2 Reverse & GTAGCAAATACATATCTGGTTGTCTTCA \\
\hline Site-directed mutagenesis primers \\
\hline PGRMC1 S57/A Forward & CAGCGGCGACgccGACGACGACGAGCCGC \\
\hline PGRMC1 S57/D Forward & CAGCGGCGACgccGACGACGACGAGCCGC \\
\hline PGRMC1 S57/E Forward & CAGCGGCGACgagGACGACGACGAGCCGCCCC \\
\hline PGRMC1 S57 Reverse & GCCGCCGGCTGGTCCCCG \\
\hline
\end{tabular}

\section{Figures}


A

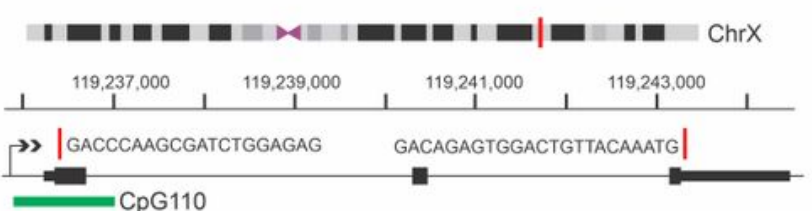

B
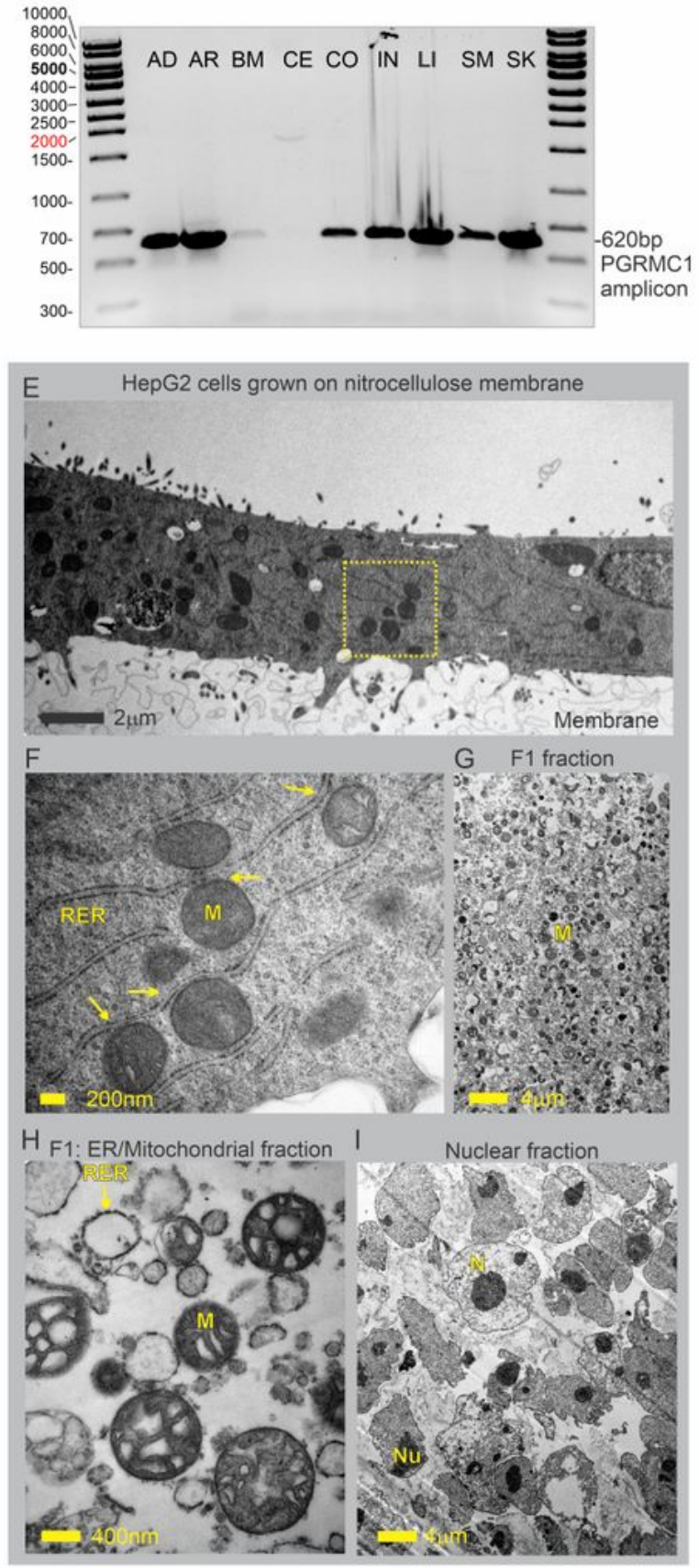

C

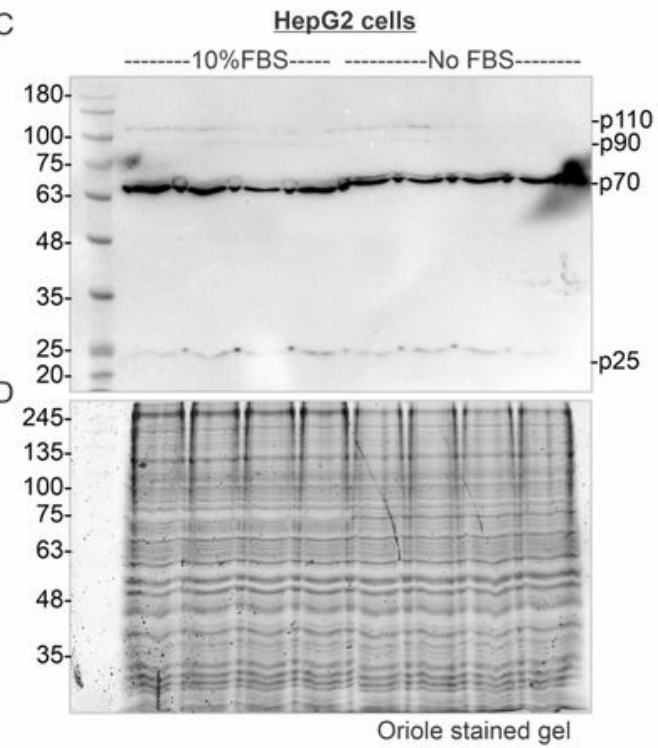

HepG2 subcellular factions

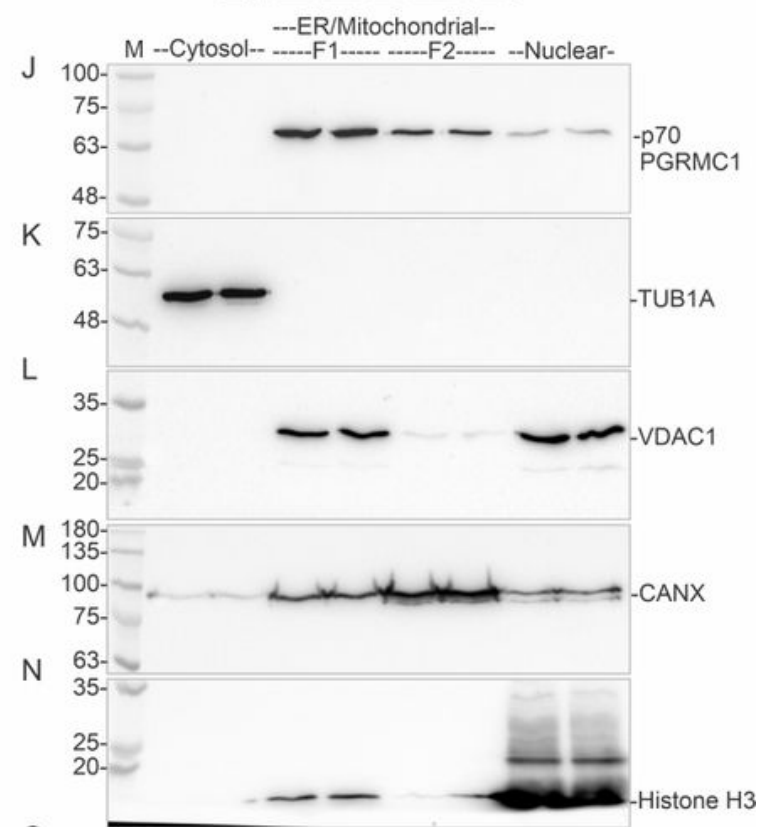

$\mathrm{O}$

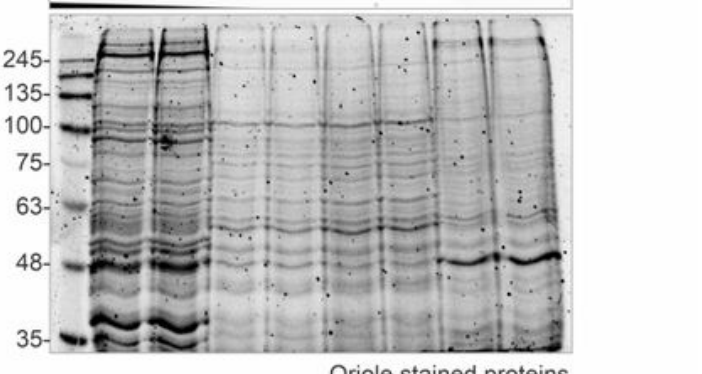

Figure 1

PGRMC1 expression in human tissues and subcellular distribution of PGRMC1 in HepG2 cells. (A): Diagrammatic representation of the human X chromosome (Chr.) showing a magnified view of the Xq24 region harboring the PGRMC1 gene. The exons are depicted in black rectangles andthe location of the RTPCR primers to amplify the full-length ORF of the PGRMC1 gene is provided. CpG: CpG Island. (B): Agarose gel image showing long-range RT-PCR-based amplification of PGRMC1 in different primary adult 
human tissues. AD: adipose; AR: artery; BM: bone marrow; CE: Cerebellum; CO: cortex; IN: intestine; LI: liver; SM: skeletal muscle; SK: skin. cDNAs equivalent to 100ng total RNA wereemployed in each PCR reaction using 35 cycles. DNA ladders in base pairs. (C): Immunoblot showing expression of PGRMC1 in HepG2 cells grown in media supplemented with or without fetal bovine serum (FBS). (D): Oriole-stained gel showing total protein loading in C. (E-F): TEM images showing the ultrastructure of HepG2 cells grown on a nitrocellulose membrane. $\mathrm{F}$ represents a higher magnification image of the yellow rectangle area in E. RER: rough endoplasmic reticulum; M: mitochondrion; Yellow arrows: mitochondria-associated ER membranes. (G) TEM image of the F1 fraction from HepG2 cells. $(\mathrm{H})$ : Higher magnification view of the RER and mitochondrial structures present in the F1 fraction. (I): TEM image of the nuclear fraction derived from HepG2 cells after mild detergent treatment. Note the absence of any visually discernible mitochondria/ER structures in the nuclear fraction. N: nucleus; Nu: nucleolus. (J-N): Immunoblots showing the presence of PGRMC1 (J), TUB1A (K), VDAC1 (L), CANX (M), and Histone H3 (N) in different subcellular fractions derived from HepG2 cells. O: Oriole-stained gel showing total protein loading in J-N. The distinct protein profiles of the subcellular fractions indicates the efficiency of the organelle enrichment process. 
A

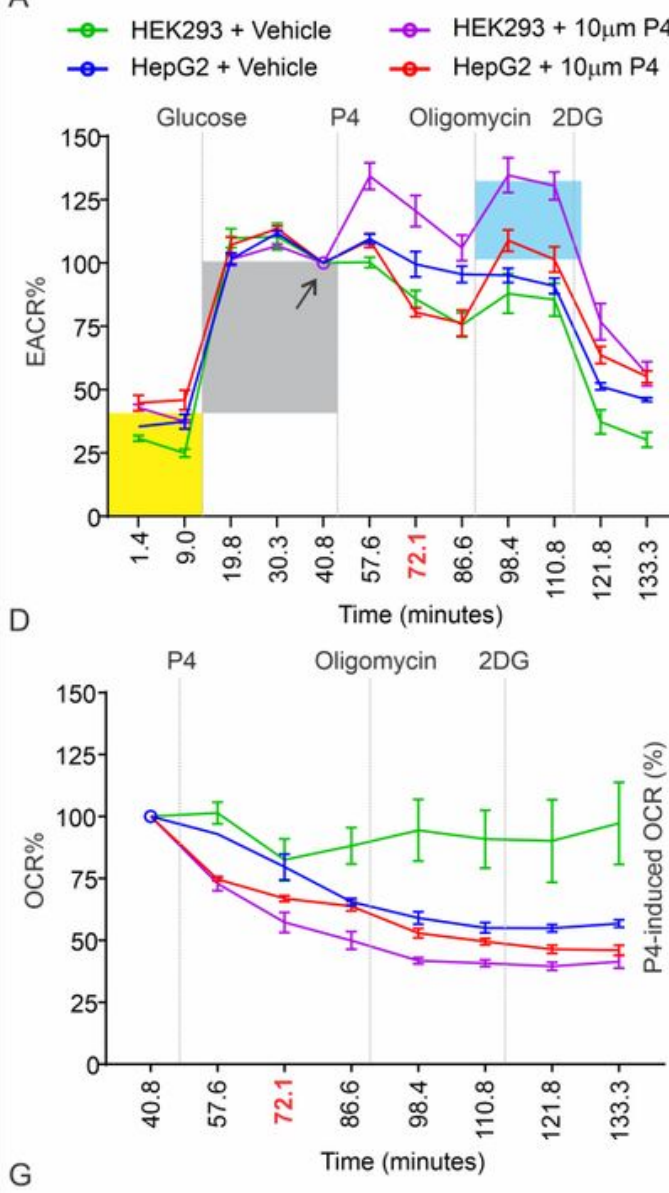

G

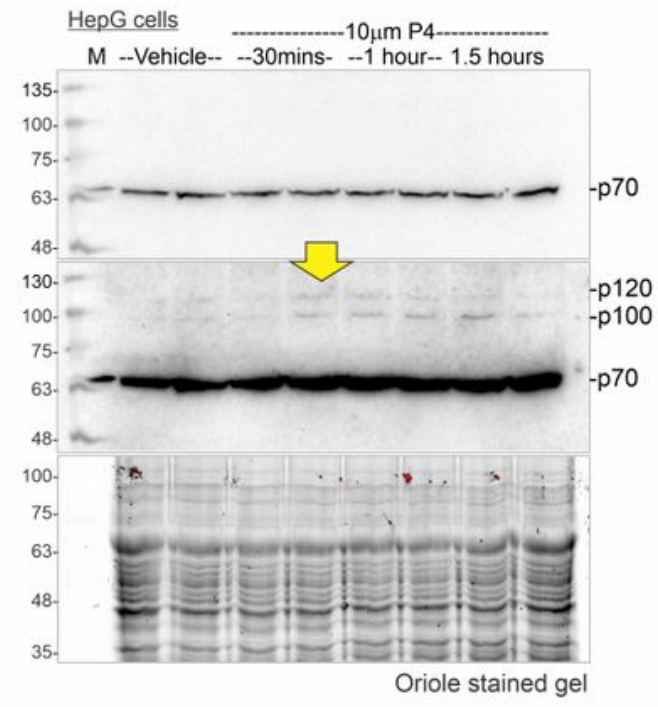

B

HepG2 HEK293

C

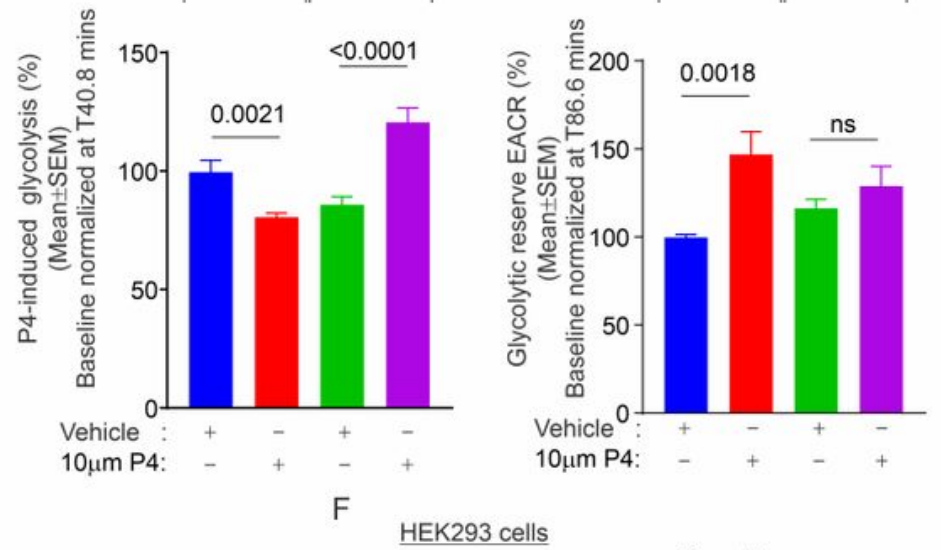

E

E

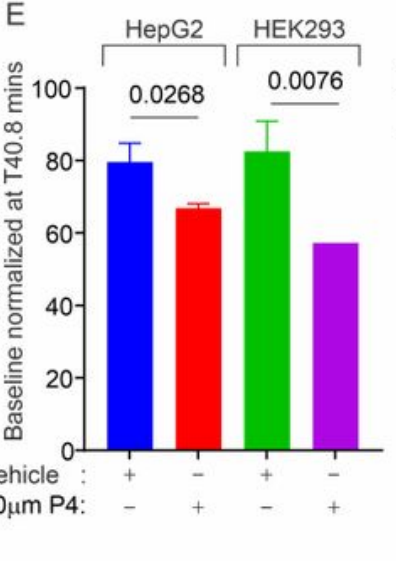

M --Vehicle-- -30 mins- -1 hour-- -1.5 hours-

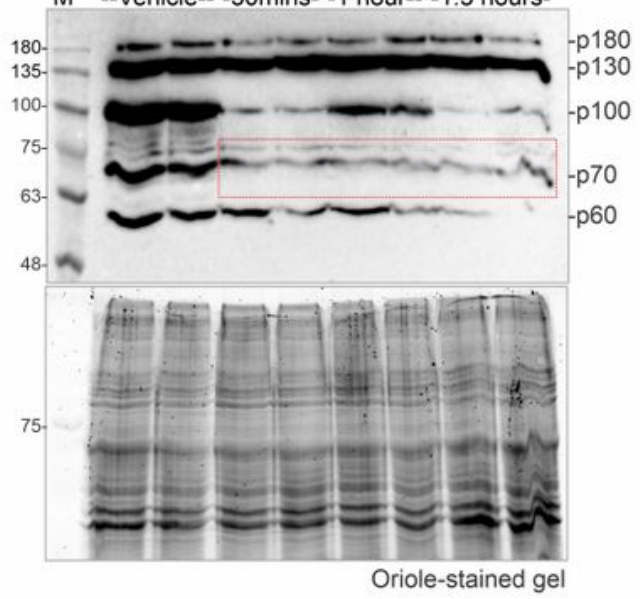

$\mathrm{H}$

I

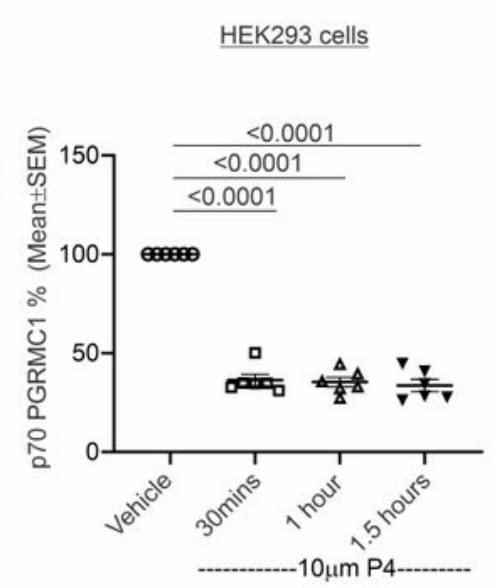

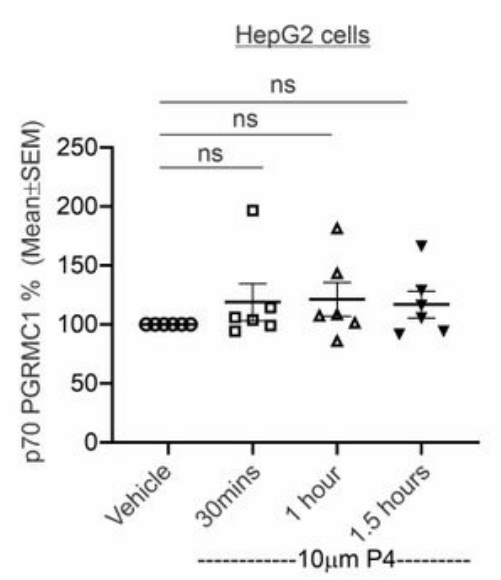

Figure 2

Glycolysis stress test showingcell-type-specific P4-induced glycolysis in HEK293 and HepG2 cells. (A\&D): Line graphs showing ECAR (A) and OCR (D) following sequential injection of glucose, P4, oligomycin, and 2DG (2-deoxyglucose). The final concentrations of the compounds are as follows: Glucose (10mM), P4 $(10 \mu \mathrm{M})$, Oligomycin $(1 \mu \mathrm{M})$, and 2DG $(50 \mathrm{mM})$. Each data point represents mean $\pm S E M$. $\mathrm{N}=5$ replicates. The readings (at time point 72.1 mins) were normalized based on the basal values before P4 injection (black 
arrow). Non-glycolytic acidification (yellow-colored rectangle): last rate measurement before glucose injection. Glycolysis (grey rectangle): maximum rate measurement before oligomycin injection minus last rate measurement before glucose injection. Cyan rectangle: glycolytic reserve volume. (B-C \&E): Bar graphs showing P4-induced glycolysis (B), glycolytic reserve (C), and P4-induced respiration (E). N=10 from two independent experiments. Data were analyzed by one-way ANOVA followed by Dunnett's post hoc multiple comparison test. $p$ values are shown for the post hoc tests. (F-G): Immunoblots showing PGRMC1 levels in the vehicle and $10 \mathrm{LM}$ P4-treated HEK293 (F) and HepG2 (G) cells at different time points. The oriole-stained gel image in the bottom panel is showing total protein loading. The middle panel in $\mathrm{G}$ is a longer exposure of the blot. $(\mathrm{H}-\mathrm{I})$ : Scatter plots showing the relative amount of PGRMC1 in the vehicle and P4-treated HEK293 (H) and HepG2 (I) cells at different time points. $\mathrm{N}=6$ from two independent experiments. Data were analyzed by one-way ANOVA followed by Dunnett's post hoc multiple comparison test; $p$ values are shown for the multiple comparison tests; ns: not significant $(p>0.05)$. 
A
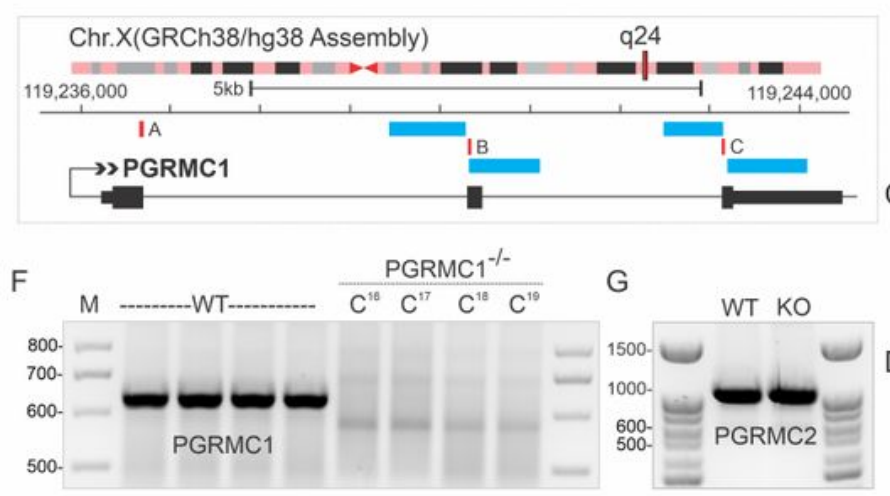

$\mathrm{H}$

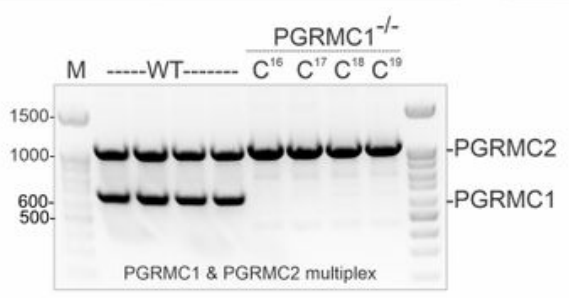

B M WT WT $\frac{\text { PGRMC1 } 1^{-1-} \text { Clones }}{C^{13} C^{14} C^{15} C^{18} C^{17} C^{18} C^{19}}$

Fig-3

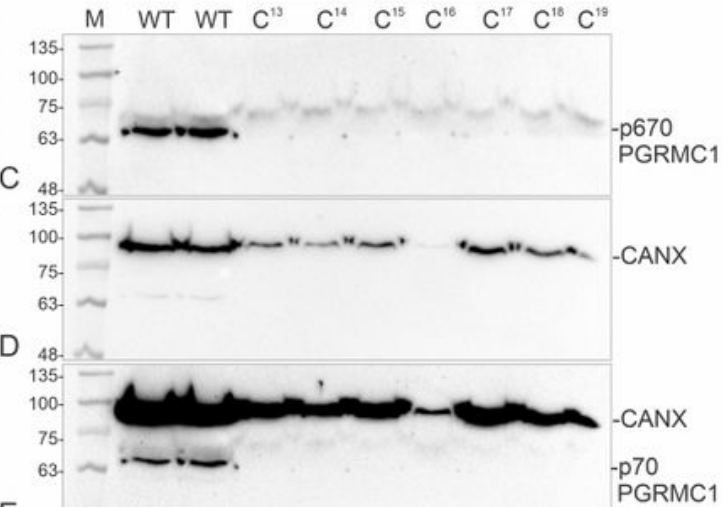

E

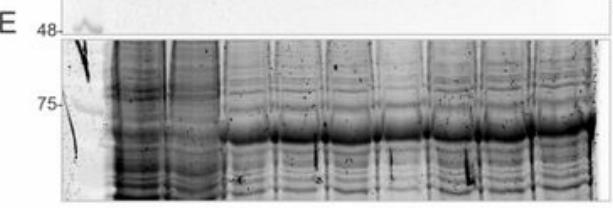

Oriole stained protein profile
I
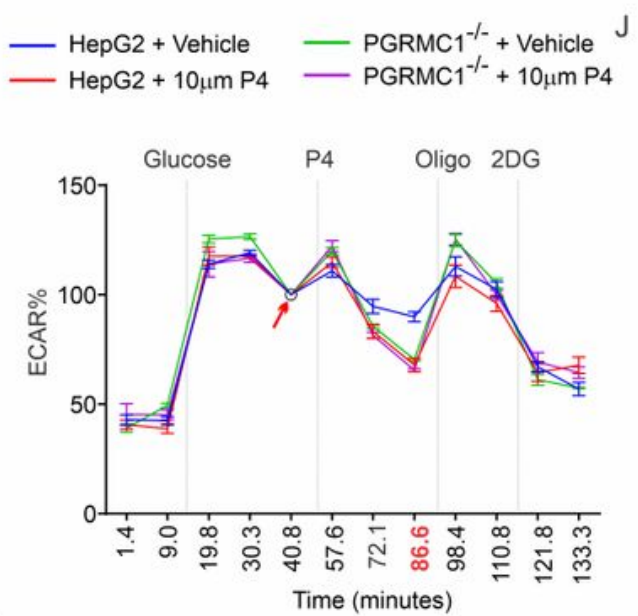

K

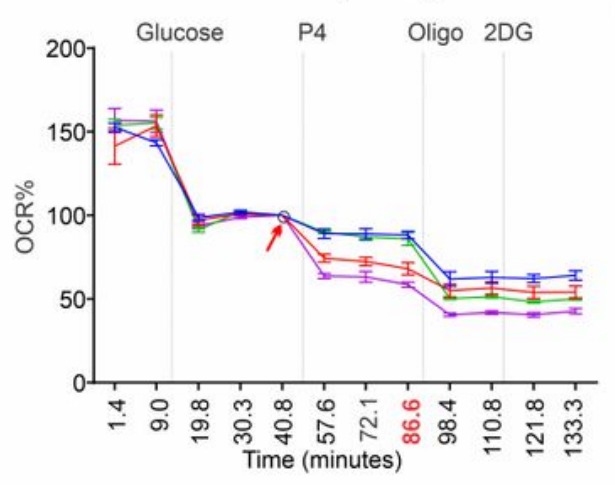

- HepG2 + Vehicle $=\mathrm{PGRMC1}^{-1-}+$ Vehicle

- HepG2 + 10um P4 $=\mathrm{PGRMC1}^{-1-}+10 \mu \mathrm{m} \mathrm{P4}$

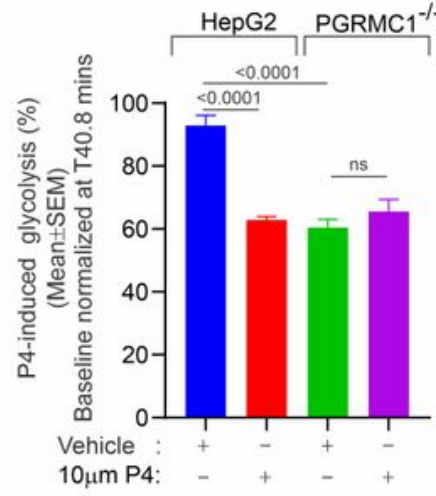

L

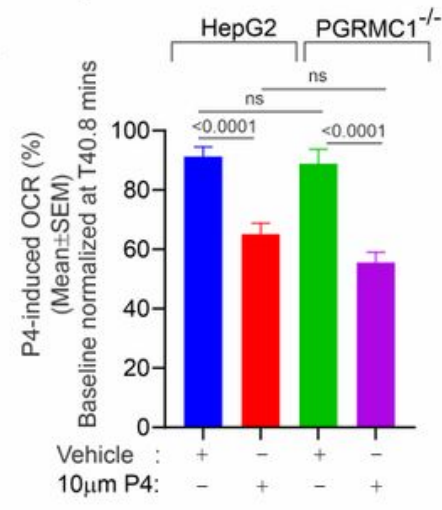

\section{Figure 3}

Glycolysis stress test showing P4-induced glycolysis in PGRMC1-/-and wild-type HepG2 cells. (A):

Diagram showing the genomic location of two PGRMC1 specific gRNA sequences and the reporter/selection cassette flanked by the homology-driven repair (HDR) sequences. The black rectangles represent exons and the blue rectangles represent alignment of HDR sequences. B: Immunoblots showing loss of PGRMC1 expression in multiple HepG2 cell clones generated by CRISPR/Cas9-mediated deletion 
of the PGRMC1 gene. (C-D): CANX expression in the immunoblot presented in B. (E): Oriole-stained gel showing total protein loading. (F-G): Agarose gel image showing amplification of the full-length PGRMC1 (F) and PGRMC2 (G) ORFs in different wild-type and PGRMC1 knockout (KO) HepG2 cell clones. cDNAs equivalent to 100ng total RNA were employed in each PCR reaction using 35 cycles. DNA ladders in base pairs. $(H)$ Multiplex PCR showing co-amplification of PGRMC1 and PGRMC2 in the wild-type and PGRMC1-deleted HepG2 cell clones. (I\&K): Line graphs showing ECAR (I) and OCR (K) following sequential injection of glucose, P4, oligomycin, and 2DG. Oligo: Oligomycin, 2DG: 2-deoxyglucose. The final concentrations of the compounds are as follows: Glucose $(10 \mathrm{mM}), \mathrm{P} 4(10 \mu \mathrm{M})$, Oligomycin $(1 \mu \mathrm{M})$, and $2 \mathrm{DG}(50 \mathrm{mM})$. Each data point represents mean \pm SEM. $\mathrm{N}=5$ replicates. The percentages (at time point 86.6 mins) were normalized based on the basal values before P4 injection (red arrows). (J\&L): Bar graphs showing P4-induced glycolysis $(\mathrm{J})$ and respiration $(\mathrm{L})$. $\mathrm{N}=10$ from two independent experiments. Data were analyzed by one-way ANOVA followed by Dunnett's post hoc multiple comparison test. $p$ values are shown for the multiple comparison tests. 
A

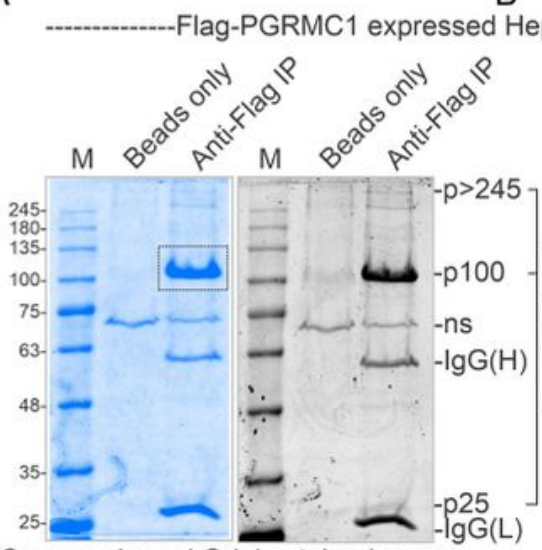

Coomassie and Oriole stained mouse anti-Flag immunoprecipitate (IP)

C

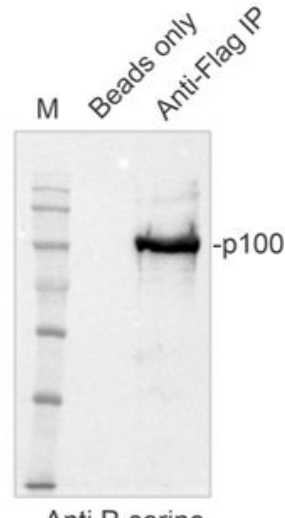

Anti P-serine

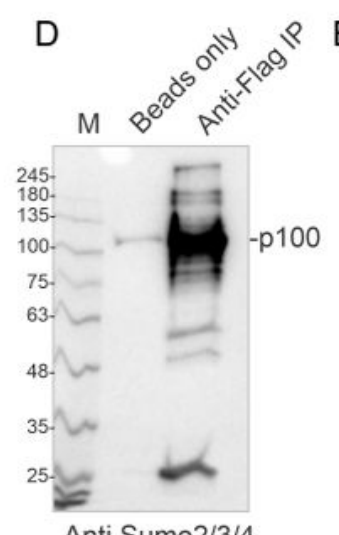

Anti Sumo2/3/4

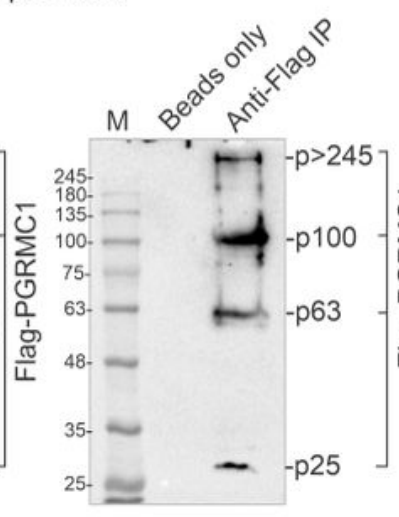

Rabbit Anti-Flag

I

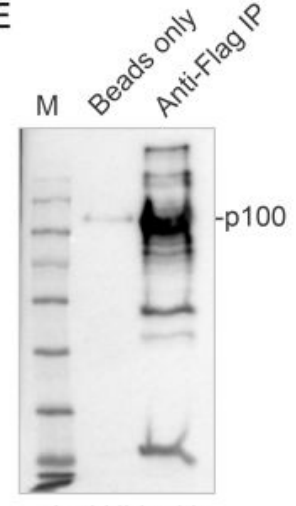

Anti Ubiquitin
$\mathrm{F}$

Fig-4
G

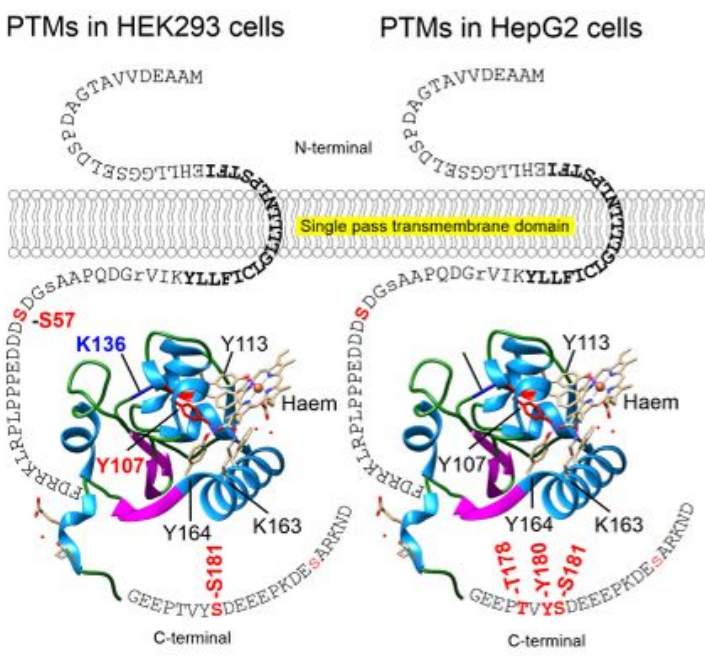

\begin{tabular}{|c|c|c|c|c|c|c|c|}
\hline Spectrum & $\log (e)$ & $\mathrm{m}+\mathrm{h}$ & Modifcation & Start & PGRMC1 peptide sequence & End & \\
\hline 15593.1 & -12 & 2730.203 & $\mathrm{P}-\mathrm{Y} 180$ & 170 & LLKEGEEPTVYSDEEEPKDESAR & 192 & 54511 \\
\hline 15593.2 & -12 & 2730.203 & $\mathrm{P}-\mathrm{S} 181$ & 170 & LLKEGEEPTVYSDEEEPKDESAR & 192 & 54511 \\
\hline 15475.1 & -7.9 & 2730.203 & $\mathrm{P}-\mathrm{T} 178$ & 170 & LLKEGEEPTVYSDEEEPKDESAR & 192 & 54511 \\
\hline 15475.2 & -7.9 & 2730.203 & $\mathrm{P}-\mathrm{Y} 180$ & 170 & LLKEGEEPTVYSDEEEPKDESAR & 192 & 54511 \\
\hline 15475.3 & -7.9 & 2730.203 & $\mathrm{P}-\mathrm{S} 181$ & 170 & LLKEGEEPTVYSDEEEPKDESAR & 192 & 54511 \\
\hline 15500.1 & -3.7 & 2730.203 & $\mathrm{P}-\mathrm{Y} 180$ & 170 & LLKEGEEPTVYSDEEEPKDESAR & 192 & 54511 \\
\hline 15500.2 & -3.7 & 2730.203 & $\mathrm{P}-\mathrm{S} 181$ & 170 & LLKEGEEPTVYSDEEEPKDESAR & 192 & 54511 \\
\hline 12870.1 & -5.6 & 2858.298 & $\mathrm{P}-\mathrm{Y} 180$ & 170 & LLKEGEEPTVYSDEEEPKDESARK & 193 & 5106 \\
\hline 12870.2 & -5.6 & 2858.298 & $\mathrm{P}-\mathrm{S} 181$ & 170 & LLKEGEEPTVYSDEEEPKDESARK & 193 & 5106 \\
\hline \multicolumn{8}{|c|}{ PGRMC1 Peptides previously found phosphorylated/actylated in HEK293 cells } \\
\hline 15418.1 & -15 & 2404.13752 & non-phosphorylated S57 & 45 & IVRGDQPAASGDSDDDEPPPLPR & 67 & 60292 \\
\hline 16265.1 & -15 & 2035.88393 & non-phosphorylated S57 & 48 & GDQPAASGDSDDDEPPPLPR & 67 & 137118 \\
\hline 16283.1 & -5.8 & 2035.88393 & non-phosphorylated 557 & 48 & GDQPAASGDSDDDEPPPLPR & 67 & 137118 \\
\hline 27512.1 & -15 & 1516.72194 & non-phosphorylated Y107 & 106 & FYGPEGPYGVEAGR & 119 & 61073 \\
\hline 29817.1 & -13.9 & 4629.15012 & non-acetylated $\mathrm{K}-136$ & 124 & GLATFCLDKEALKDEYDDLSDLTAAQQETLSDWESQFTFK & 163 & 1704 \\
\hline 29643.1 & -15 & 3624.639 & non-acetylated $\mathrm{K}-136$ & 133 & EALKDEYDDLSDLTAAQQETLSDWESQFTFK & 163 & 13649 \\
\hline
\end{tabular}

Figure 4

Mass spectrometric characterization of PGRMC1 PTMs in HepG2 cells. (A): Coomassie and oriole stained gel showing anti-Flag immunoprecipitated proteins from Flag-PGRMC1 transiently expressed HepG2 cells. IP: Immunoprecipitate; Beads only: Dynabeads only; ns: nonspecific. (B): Immunoblot showing the presence of Flag-PGRMC1 in the anti-Flag immunoprecipitate. (C-E): Immunoblots showing the presence of anti-P-Serine, anti-SUM02/3/4, and anti-ubiquitin positive bands corresponding to Flag-PGRMC1 
proteins in HepG2 cells. (F): A comparison of the PGRMC1 PTMs identified in HEK293 and HepG2 cells. The PGRMC1 PTMs in HEK293 cells were identified in a previous study[3]. (G\&H): Crystal structures showing PGRMC1 cytochrome b5-like domain bound to haem and the $\mathrm{N}$ and $\mathrm{C}$ terminal sequences in HEK293 (G) and HepG2 (H) cells. The cell-type-specific differential post-translationally modified residues (bold and red/blue) of PGRMC1 are highlighted in the crystal structure. Adapted from PDB ID: 4X8Y[26]. The $\beta$-strands and the a-helices are highlighted in pink and blue colors, respectively. The Tyr113, Tyr107, Lys163, and Tyr164 are the haem-binding residues[26]. The P-S57 is localized in the N-terminal region and the P-T178, P-Y180 and S181 residues are in the C-terminal region. The $\mathrm{N}$-terminal transmembrane motif is highlighted in bold. (I) Table showing representative MS/MS spectrums of the PGRMC1 posttranslationally modified peptides identified in HepG2 cells. The spectrum is written in the form "X.Y", where $X$ is a unique identifier for a particular tandem mass spectrum in this data set and $Y$ is an identifier for this particular sequence solution. $\log (\mathrm{e})$ : the base-10 log of the expectation that any particular peptide assignment was made at random (E-value). $m+h$ : the calculated mass of the protonated parent ion for this sequence assignment.Sequence: the sequence of the assigned peptide sequence. $n$ : the number of observations of the respective peptide sequence in the Global Proteome Machine Database (GPMDB)[92]. The modified residues in the peptides are highlighted in red. 
-Flag-PGRMC1 expressed HepG2 cells
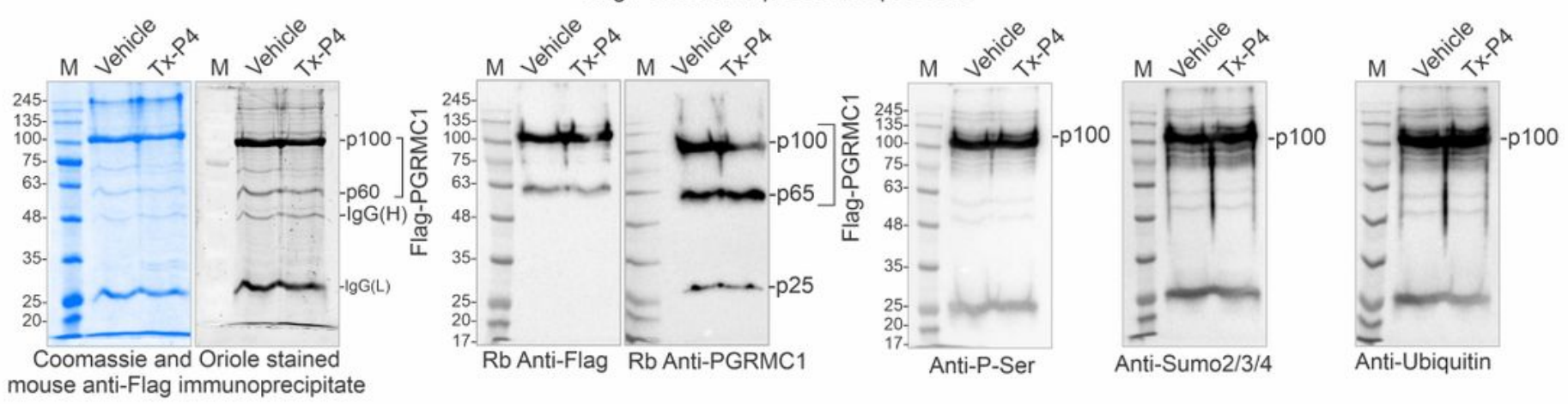

HEK293 and HepG2 cell-based (IEF/SDS-PAGE analysis)

G
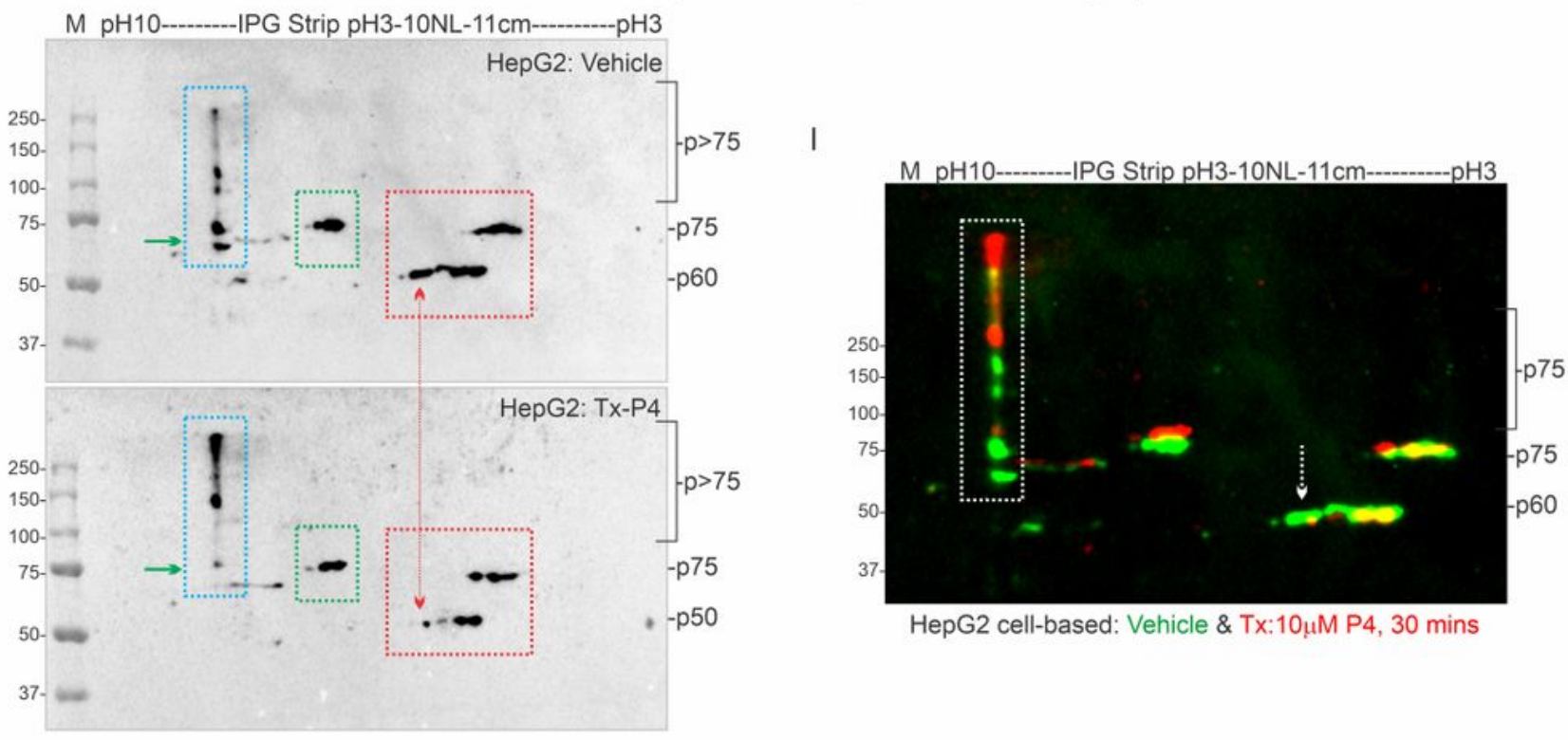

HepG2 cell-based: Vehicle \& Tx:10 $\mu \mathrm{M}$ P4, 30 mins

J

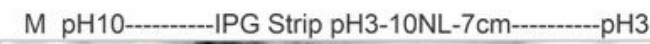

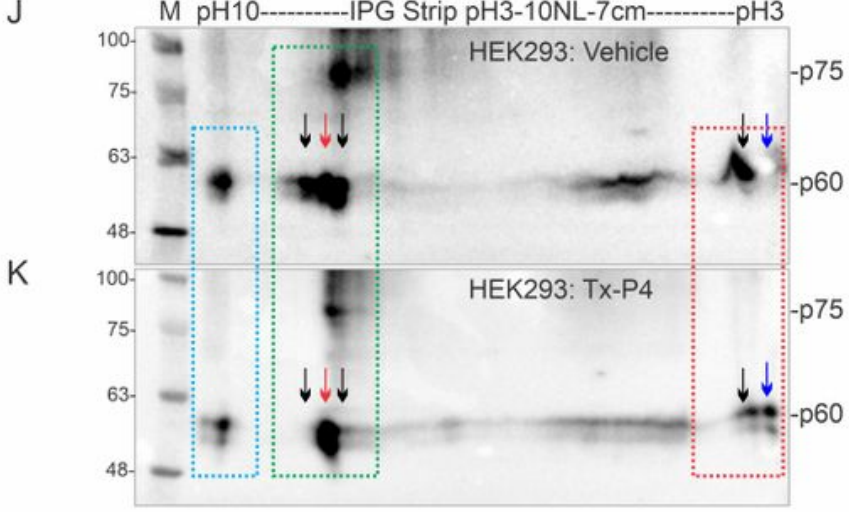

$\mathrm{L}$

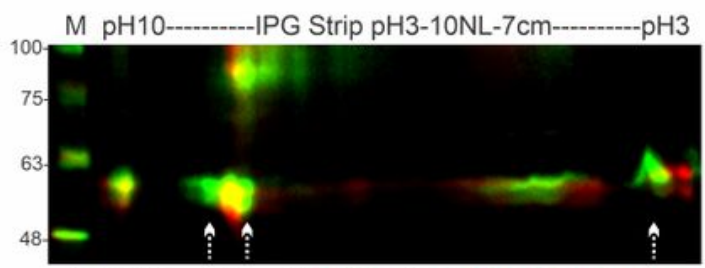

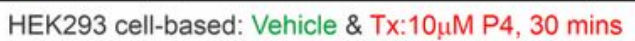

\section{Figure 5}

P4-induced cell type-specific differential PTMs (charged fractions) of PGRMC1. (A): Coomassie and oriole-stained gel showing anti-Flag immunoprecipitated proteins from Flag-PGRMC1 transiently expressed vehicle (Ethanol) and P4-treated HepG2 cells. Tx-P4: Treated with $10 \square \mathrm{M} \mathrm{P4}$ for 30 mins. (B-C): Immunoblot showing the presence of Flag-PGRMC1 in the anti-Flag immunoprecipitate. (D-F): Immunoblots showing the presence of P-serine (D), SUMO2/3/4 (E), and Ubiquitin (F) positive bands 
corresponding to Flag-PGRMC1 p100 proteins in HepG2 cells. (G-H): Immunoblots showing charged fractions (PTMs) of PGRMC1 protein in the vehicle $(\mathrm{G})$ and P4-treated $(\mathrm{H}) \mathrm{HepG} 2$ cells. The red and green arrows representa differential shift of the focused spots. Blue rectangle: $\mathrm{pl} / \mathrm{pH} 8-9$; green rectangle: $\mathrm{pl} / \mathrm{pH}$ 6-7; red rectangle: $\mathrm{pl} / \mathrm{pH} 5-6$. (I): The immunoblots in $\mathrm{G} \& \mathrm{H}$ were false-colored and overlaid to show the relative shift of the PGRMC1 charged fractions (white arrow and rectangle). (J\&K):

Immunoblots showing charged fractions of PGRMC1 protein in the vehicle (J) and P4-treated (K) HEK293 cells. Colored arrows representa differential shift of the focused spots. Blue rectangle: $\mathrm{pl} / \mathrm{pH} 9-10$; green rectangle: $\mathrm{pl} / \mathrm{pH} 8-9$; red rectangle: $\mathrm{pl} / \mathrm{pH} 3-4$. (L): The immunoblots in J\&K were false-colored and overlaid to show the relative shift of the PGRMC1 charged fractions (white arrows). 


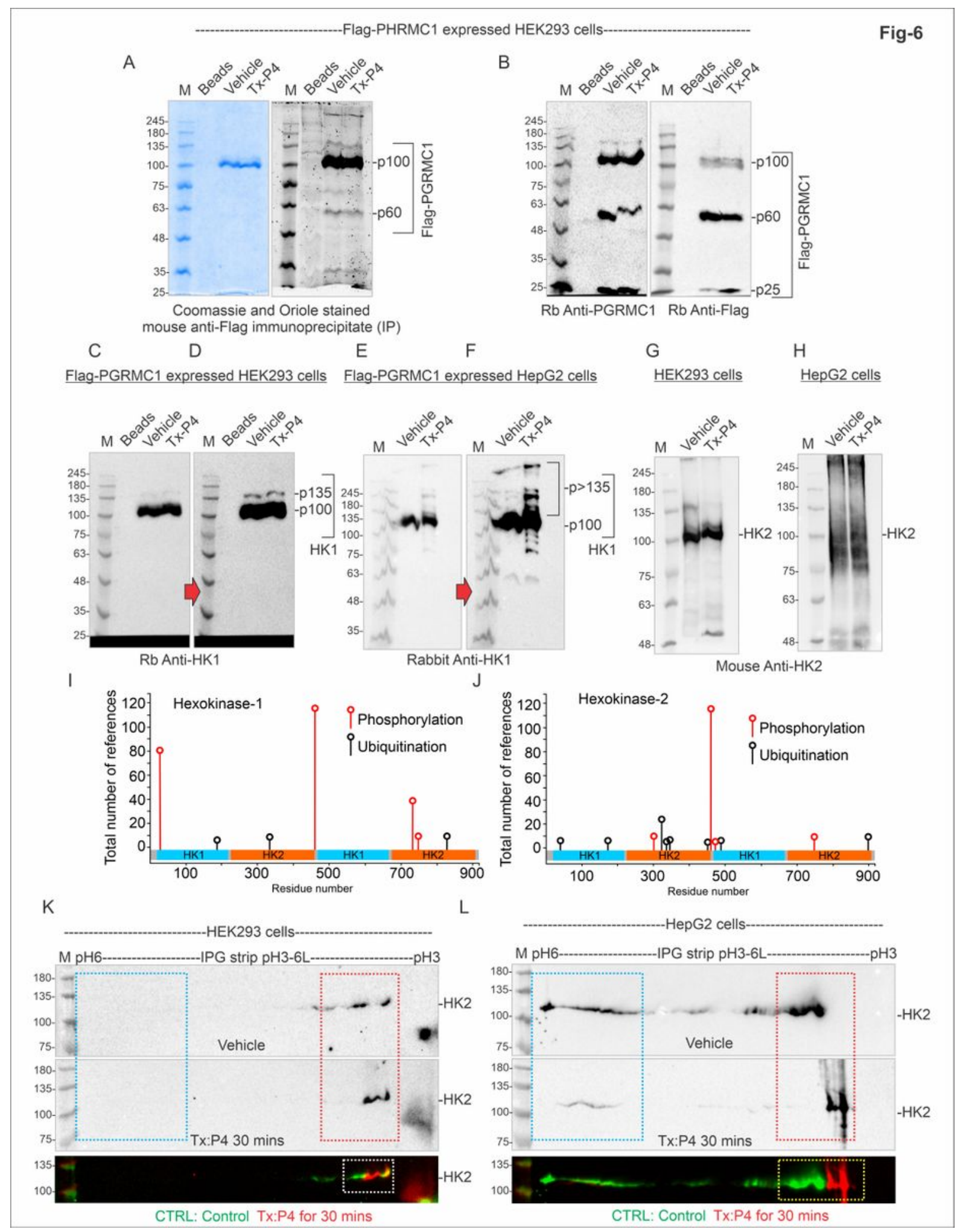

\section{Figure 6}

Differential interaction of PGRMC1 with HK1/2 and the effect of P4 on HK2 PTMs in HEK293 and HepG2 cells. (A): Coomassie and oriole-stained gel showing anti-Flag immunoprecipitated proteins from FlagPGRMC1 transiently expressed vehicle (Ethanol) and P4-treated HEK293 cells. The IgG was crosslinked to the protein G using BS3, therefore, corresponding bands are not present. Beads: Dynabeads only; Tx-P4: Treated with $10 \square \mathrm{M}$ P4 for 30 mins. (B): Immunoblot showing the presence of Flag-PGRMC1 in the anti- 
Flag immunoprecipitate. (C-F): Immunoblots showing the presence of HK1 in anti-Flag immunoprecipitated proteins from Flag-PGRMC1 transiently expressed vehicle (Ethanol) and P4-treated HEK293 (C\&D) and HepG2 (E\&F) cells. Red arrows indicate higher exposure of the respective immunoblots to highlight under-exposed bands. (G\&H): Immunoblots showing presence/absence of HK2 in anti-Flag immunoprecipitated proteins from Flag-PGRMC1 transiently expressed vehicle (Ethanol) and P4-treated HEK293 (G) and HepG2 (H) cells. (I-J): Diagrammatic representation showing the location of different phosphorylated (red) and ubiquitinated (black) residues in HK1 and HK2 proteins identified by mass spectrometry. Colored rectangles represent functional domains. The vertical lines with circle tips represent the PTM residues and the length of the line corresponds to the number of literature references. Only PTMs with a minimum of 5 references were considered. The figure was prepared using data obtained from PhosphositePlus (www.phosphositeplus.org)[61]. (K\&L): Immunoblots showing charged fractions of HK2 protein in the vehicle (top panel) and P4-treated (middle panel) HEK293 (K) and HepG2 (L) cells. Blue rectangle: $\mathrm{pl} / \mathrm{pH} \mathrm{8-10;} \mathrm{red} \mathrm{rectangle:} \mathrm{pl} / \mathrm{pH} 4-5$. Bottom panel: The immunoblots in the top panels were false-colored and overlaid to show the relative shift of the HK2 charged fractions (white and yellow rectangles). 


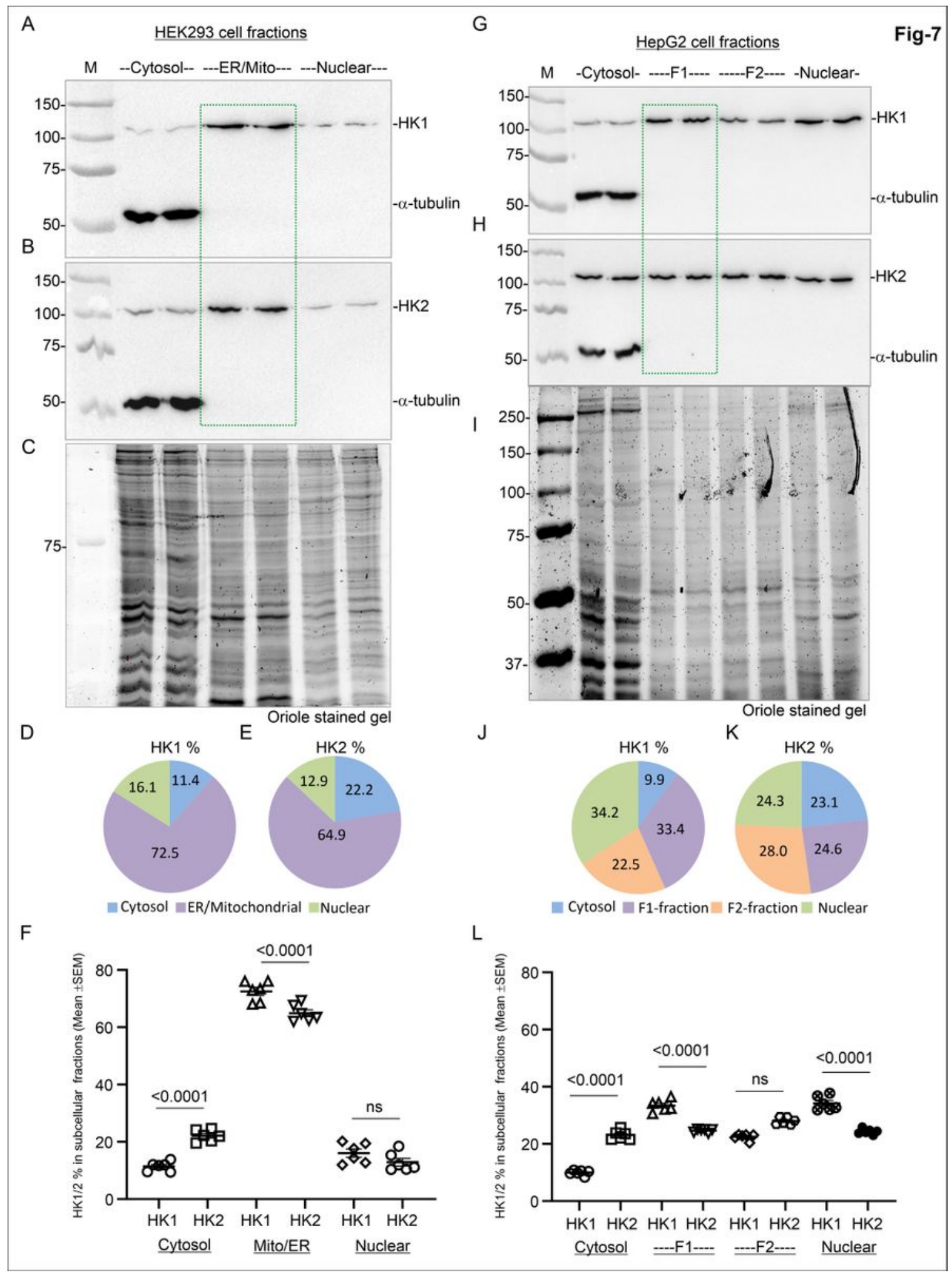

Figure 7

Subcellular distribution of HK1 and HK2 in HEK293 and HepG2 cells. (A\&B and G\&H): Immunoblots showing the presence of HK1/HK2 and a-tubulin (TUB1A) in different subcellular fractions derived from HEK293 (A\&B) and HepG2 (G\&H) cells. Green rectangle: ER/mitochondrial fraction. (C\&I): Oriole-stained gels showing total protein loadings. (D-F): Pie diagrams (D\&E) and scatter plot (F) showing the relative amount of HK1 and HK2 in different subcellular fractions in HEK293 cells. The relative amount of HKs in 
different subcellular fractions is presented as a percent of the total HKs intensity (cytosol + ER/Mitochondria+nucleus) in a single immunoblot. (J-L): Pie diagrams (J\&K) and scatter plot (L) showing the relative amount of HK1 and HK2 in different subcellular fractions in HepG2 cells. The Pie diagrams are based on the mean values presented in the scatter plots in $\mathrm{F} \& \mathrm{~L} . \mathrm{N}=6$ replicates from 3 independent experiments. Data were analyzed by one-way ANOVA followed by Dunnett's post hoc multiple comparison test. $\mathrm{p}$ values are shown for the multiple comparison tests.

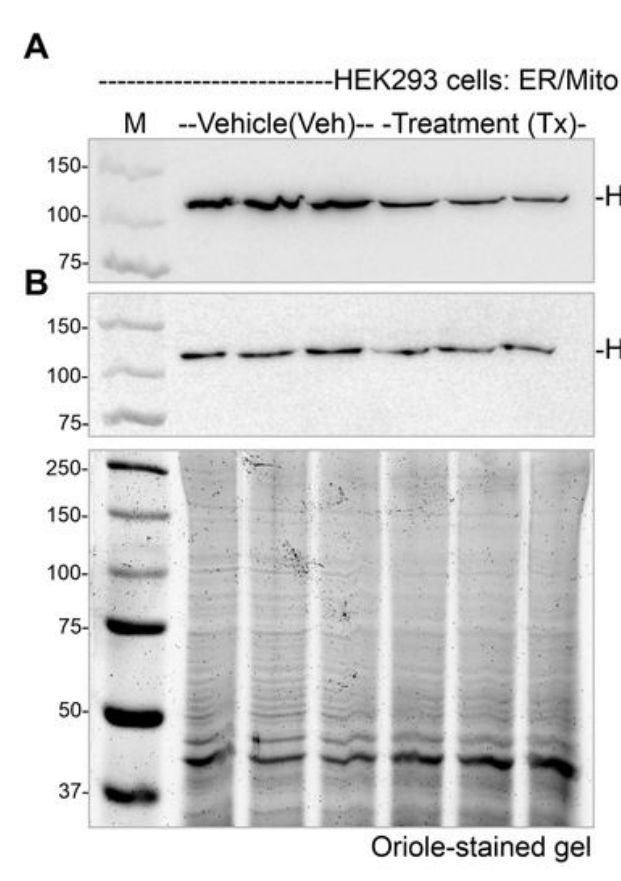

I

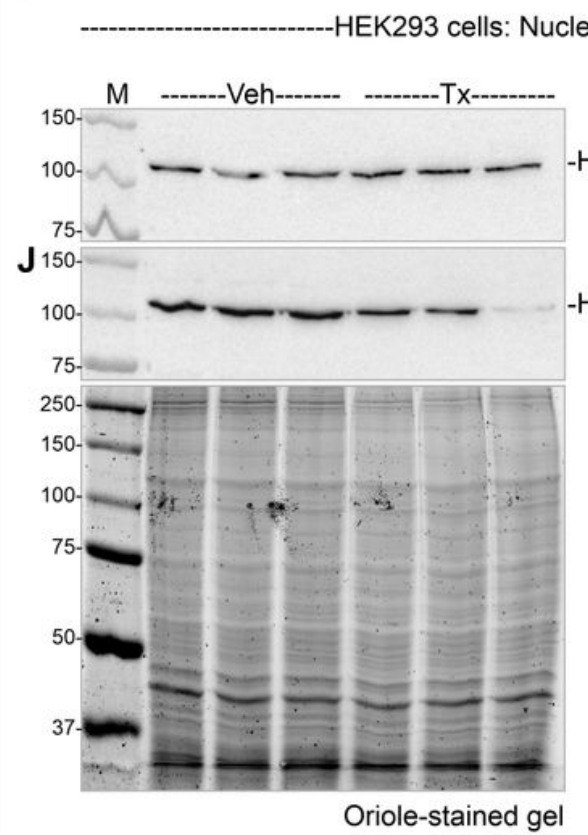

C
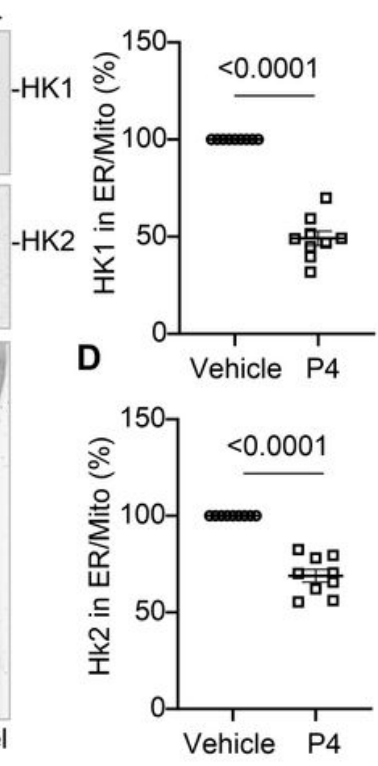

K
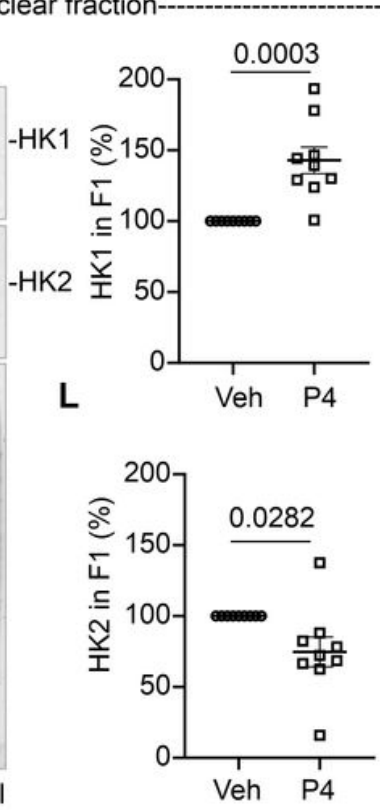

E

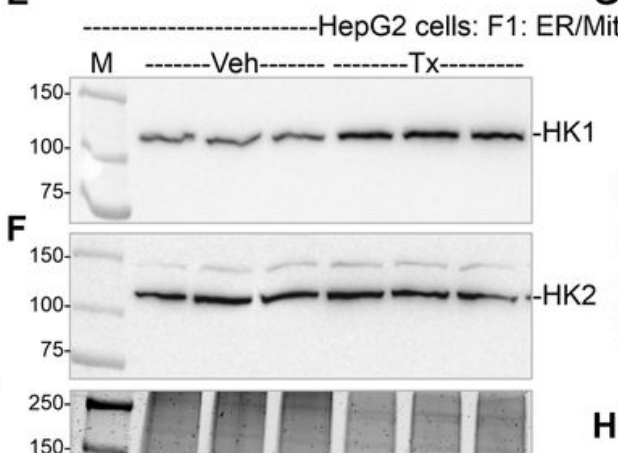

G

Fig-8

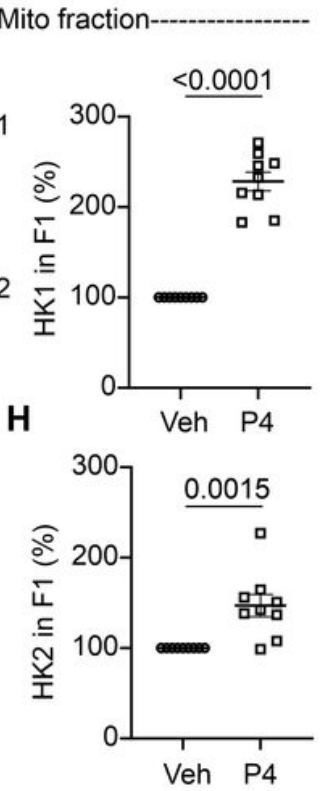

0

HepG2 cells: Nuclear fraction

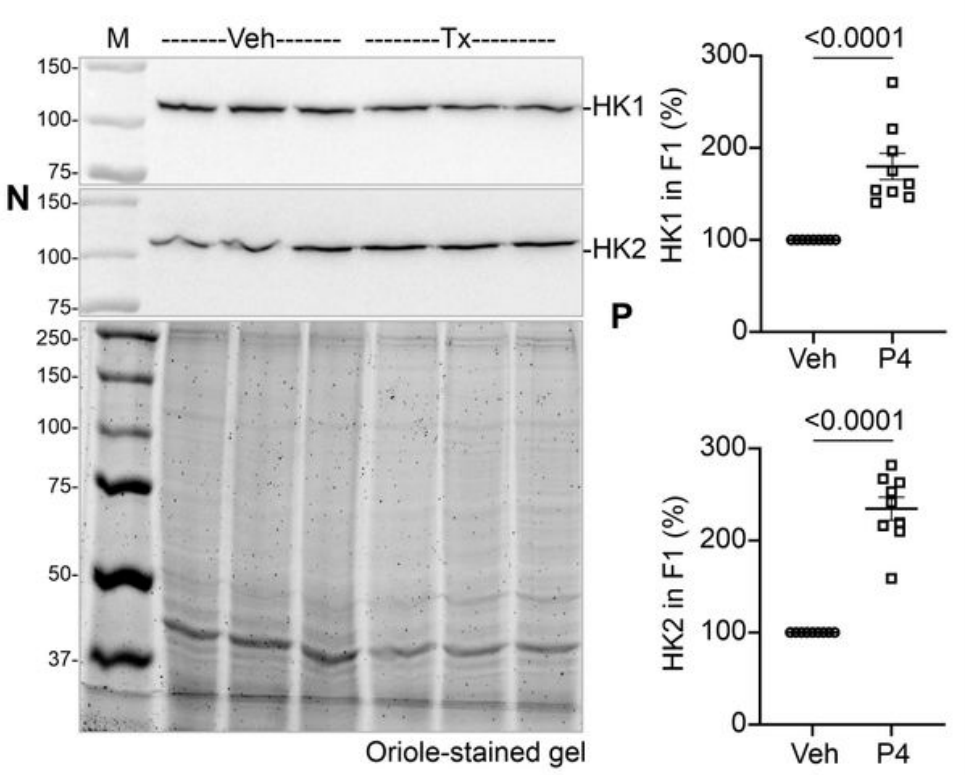

\section{Figure 8}


Effect of P4 on cell type-specific subcellular translocation of HK1 and HK2. (AB, EF, IJ, \& MN):

Immunoblots showing an abundance of HK1 and HK2 in the ER/mitochondrial fractions (F1 fractions in HepG2 cells) and nuclear fractions from the vehicle and P4-treated HEK293 and HepG2 cells.Veh: vehicle; Tx: Treated with $10 \square M$ P4 for 30 mins. (CD, GH, KL, \& OP): Scatter plots showing the relative abundance of HK1 and HK2 in the ER/mitochondrial fractions (F1 fractions in HepG2 cells) and nuclear fractions from the vehicle and P4-treated HEK293 and HepG2 cells. The oriole-stained gel images in the bottom panels of $B, F, J \& N$ represent total protein loadings. $N=9$ replicates from 3 independent experiments. Pvalue by t-test (unpaired).

\section{Supplementary Files}

This is a list of supplementary files associated with this preprint. Click to download.

- graphicalabstract.jpg

- SupplementaryTable1.pdf

- FigS8.jpg

- FigS7.jpg

- FigS6.jpg

- FigS5.jpg

- FigS4.jpg

- FigS3.jpg

- FigS2.jpg

- FigS1.jpg 